ल)

1

CRREL

Traveling Wave Solutions to the Problem of Quasi-Steady Freezing of Soils

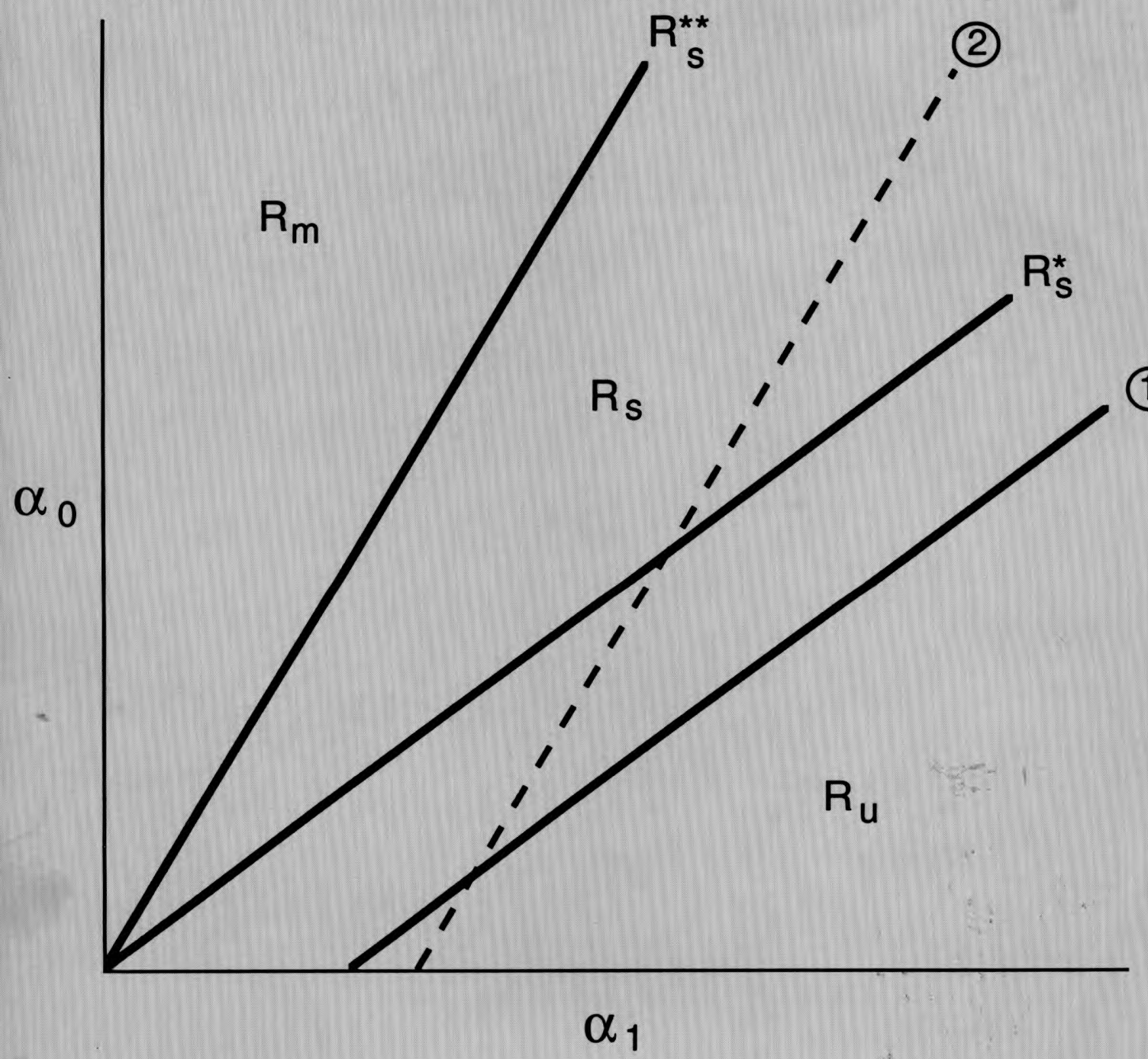




\begin{abstract}
The results of mathematical and experimental studies presented in preceding reports clearly show that the model $M_{1}$ accurately describes the properties of a frozen fringe when the steady growth of an ice layer occurs. In this work the steady growth of ice-rich frozen soil is studied by using $M_{1}$. Deriving a traveling wave solution to the problem, we have found that the condition of steady growth of ice-rich frozen soil is uniquely determined by a set of two physical variables, such as $\alpha_{0}$ and $\alpha_{1}$ used earlier, under given hydraulic conditions and overburden pressures and that the traveling wave solution converges to the solution to the problem of a steadily growing ice layer when the velocity of the $0^{\circ} \mathrm{C}$ isotherm relative to the unfrozen part of the soil vanishes.
\end{abstract}

Cover: Temperature gradients $\alpha$, and $\alpha_{0}$.

For conversion of SI metric units to U.S./British customary units of measurement consult Standard Practice for Use of the International System of Units (SI), ASTM Standard E380-89a, published by the American Society for Testing and Materials, 1916 Race St., Philadelphia, Pa. 19103. 


\section{CRREL Report 94-3}

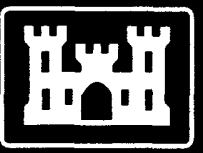

US Army Corps

of Engineers

Cold Regions Research \& Engineering Laboratory

\section{Traveling Wave Solutions to the Problem of Quasi-Steady Freezing of Soils}

Yoshisuke Nakano

March 1994 


\section{PREFACE}

This report was prepared by Dr. Yoshisuke Nakano, Chemical Engineer, of the Applied Research Branch, Experimental Engineering Division, U.S. Army Cold Regions Research and Engineering Laboratory. Funding was provided by DA Project 4A161102AT24, Research in Snow, Ice and Frozen Ground, Task SC, Work Unit F01, Physical Processes in Frozen Soil.

The author thanks Dr. Virgil Lunardini and Dr. Y.C. Yen of CRREL for their technical review of this report. 


\begin{tabular}{|c|c|}
\hline \\
\hline \multicolumn{2}{|l|}{$\begin{array}{l}\text { CONTENTS } \\
\text { Preface.......................... }\end{array}$} \\
\hline \\
\hline ...... & \\
\hline \multirow{2}{*}{\multicolumn{2}{|c|}{ (1) }} \\
\hline & \\
\hline \\
\hline \\
\hline \multicolumn{2}{|l|}{ Temperature $T(\xi)$} \\
\hline \multicolumn{2}{|l|}{ Pressure $P_{1}(\xi)$} \\
\hline \multicolumn{2}{|l|}{ Condition of steady growth } \\
\hline \multicolumn{2}{|l|}{ Discussion } \\
\hline \multicolumn{2}{|l|}{ Literature cited } \\
\hline \multicolumn{2}{|l|}{ Appendix A: Exact solution of equation 45} \\
\hline \multirow{2}{*}{\multicolumn{2}{|c|}{ Appendix B: Approximate solution of equation 45}} \\
\hline & \\
\hline $\begin{array}{l}\text { Appendix } C: \text { Computation of } I_{1} \\
\text { Abstract }\end{array}$ & \\
\hline
\end{tabular}

\section{ILLUSTRATIONS}

\section{Figure}

1. Steady growth of ice-rich frozen soil ........................................................................... 1

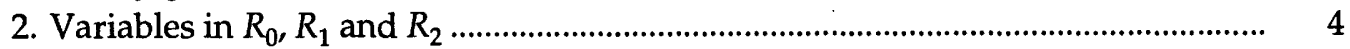

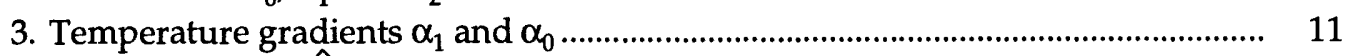

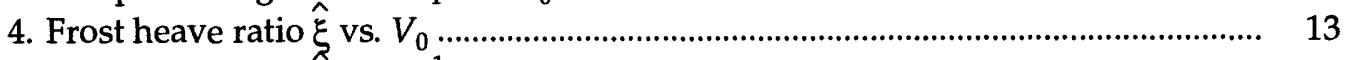

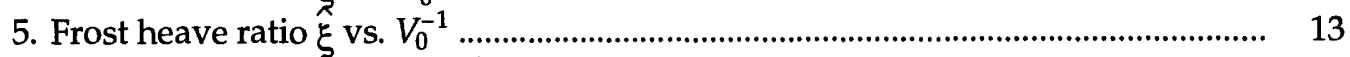

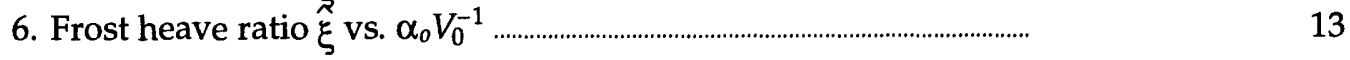




\section{NOMENCLATURE}

$a_{0} \quad$ function defined by eq $63 \mathrm{~g}$

$a_{1}$ function defined by eq $63 \mathrm{~h}$

$b$ defined by eq $63 \mathrm{c}$

$b_{0} \quad$ function defined by eq $63 \mathrm{~d}$

$b_{1}$ function defined by eq $63 \mathrm{e}$

$b_{2}$ function defined by eq $69 \mathrm{~b}$

$b_{3}$ function defined by eq $69 \mathrm{c}$

$B_{\mathrm{i}} \quad i$ th constituent of the mixture. Subscripts $i=$ 1,2 , and 3 are used to denote unfrozen water, ice and soil minerals, respectively

$c$ heat capacity of the mixture defined by eq 10c

$c_{0}$ defined by eq 41c

$c_{i}$ heat capacity of the $i$ th constituent

$d$ unit of time, day

$d_{i} \quad$ density of the $i$ th constituent

$f_{\mathrm{i}} \quad$ mass flux of the $i$ th constituent relative to that of soil minerals where $i=1,2$

$f_{\mathrm{ij}}$ mass flux of the $i$ th constituent relative to that of soil minerals in $R_{\mathrm{j}}$ where $i=1,2$ and $j=0,1,2$

$h_{\mathrm{i}} \quad$ heat content of the $i$ th constituent

$I_{0} \quad$ function defined by eq $56 \mathrm{a}$

$I_{1} \quad$ function defined by eq $56 \mathrm{~b}$

$\mathrm{I}_{2} \quad$ function defined by eq $59 \mathrm{~b}$

$k$ thermal conductivity of the mixture

$k_{\mathrm{i}} \quad$ thermal conductivity in $R_{\mathrm{i}}$ where $i=0,1,2$

$k_{11}$ limiting value of $k_{1}$ defined by eq $40 \mathrm{~h}$

$k_{21}$ limiting value of $k_{2}$ defined by eq 48

$K_{0}$ hydraulic conductivity in the unfrozen part of the soil

$K_{i}$ empirical function defined by eq 1 where $i=$ 1,2

$K_{\mathrm{i} 1}$ limiting value of $K_{\mathrm{i}}$ as $\xi$ approaches $n_{1}$ while $\xi$ is in $R_{1}, i=1,2$

$K_{i 0} \quad$ limiting value of $K_{\mathrm{i}}$ as $\xi$ approaches $n_{0}$ while $\xi$ is in $R_{1}, i=1,2$

$L \quad$ latent heat of fusion of water, $334 \mathrm{~J} \mathrm{~g}^{-1}$

$m$ boundary where the content of unfrozen water is negligible

$M_{\mathrm{i}} \quad$ name of a model defined in Part I where $i=$ $1,2,3$ $n$ boundary in $R_{0}$ and also used a generic moving surface

$\dot{n} \quad$ velocity of $n=d n / d t$

$n_{\mathrm{i}} \quad$ boundary with $i=0,1$ where $n_{0}$ denotes the boundary where $T=0^{\circ} \mathrm{C}$ and $n_{1}$ the interface between $R_{2}$ and a frozen fringe

$p_{o}$ gravity term, $0.098 \mathrm{kPa} / \mathrm{cm}$

$P_{i} \quad$ pressure of the $i$ th constituent where $i=1,2$

$P_{10}$ value of $P_{1}$ at $n_{0}$

$P_{1 \mathrm{n}}$ value of $P_{1}$ at $n$

$P_{21}$ value of $P_{2}$ at $n_{1}$

$q$ heat fluxin the mixture by conduction defined by eq $8 b$

$q^{+} \quad$ limiting value of $q$ as $\xi$ approaches $n_{1}$ while $\xi$ is in $R_{1}$

$q^{-} \quad$ limiting value of $q$ as $\xi$ approaches $n_{1}$ while $\xi$ is in $R_{2}$

$q_{i} \quad$ heat flux in the $i$ th constituent by conduction

$r$ rate of frost heave

$R_{0} \quad$ unfrozen part of the soil

$R_{1}$ frozen fringe

$R_{2} \quad$ frozen part of the soil

$R_{\mathrm{m}}$ region in the diagram of temperature gradients where an ice layer melts

$R_{\mathrm{S}} \quad$ region in the diagram of temperature gradients where the steady growth of an ice layer occurs

$R_{\mathrm{S}}^{*} \quad$ boundary between $R_{\mathrm{s}}$ and $R_{\mathrm{u}}$

$R_{\mathrm{u}} \quad$ region in the diagram of temperature gradients where the steady growth of an ice layer does not occur

$s$ defined by eq $32 \mathrm{~b}$

$s^{+} \quad$ defined by eq $31 \mathrm{~b}$

$S$ defined by eq $71 \mathrm{e}$

$S_{\mathrm{i}} \quad$ defined by eq $70 \mathrm{~d}-70 \mathrm{~g}$ where $i=1,2, \cdot, 4$

$t$ time

$T$ temperature of the mixture

$T_{i} \quad$ temperature at $n_{\mathrm{i}}$ where $i=0,1$

$T_{o} \quad$ temperature at $n_{0}$ and used also as a reference temperature

$v_{i} \quad$ velocity of the $i$ th constituent where $i=1,2,3$

$v_{\mathrm{ij}} \quad v_{\mathrm{i}}$ in $R_{\mathrm{j}}$ where $i=1,2,3$ and $j=0,1,2$ 


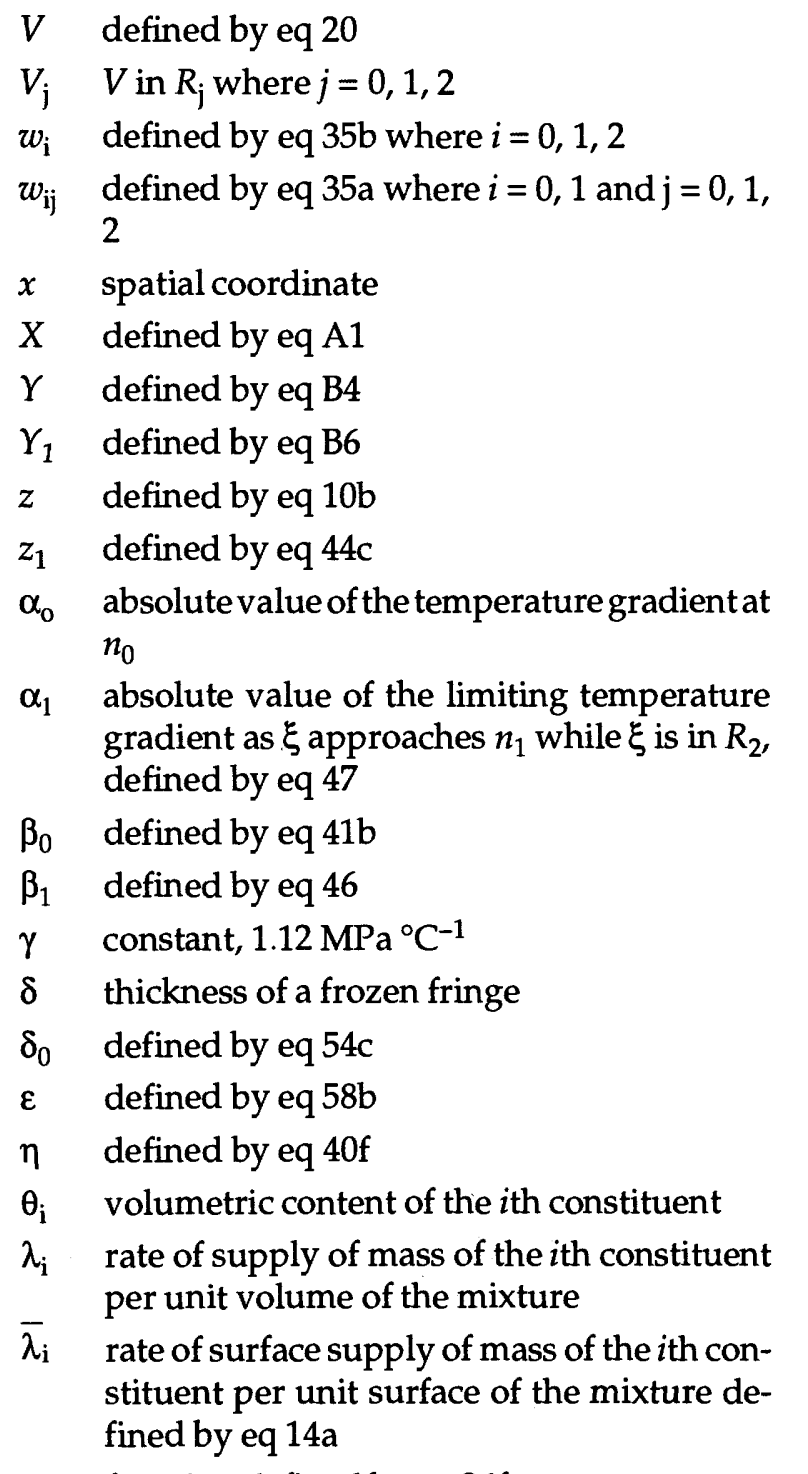

$\Lambda$ function defined by eq $26 f$
$\Lambda_{1}^{+}$function defined by eq $23 \mathrm{f}$

$\mu \quad$ defined by eq $24 \mathrm{e}$

$\mu_{0} \quad$ defined by eq $34 \mathrm{a}$

$v$ defined by eq $22 \mathrm{~h}$

$\bar{v} \quad$ defined by eq $B 8$

$v_{1} \quad$ defined by eq $23 \mathrm{i}$

$\bar{v}_{1} \quad \bar{v}$ at $T=T_{1}$

$\xi \quad$ coordinate defined by eq 16

$\xi \quad$ frost heave ratio defined by eq 72

$\pi_{0} \quad$ defined by eq B2

$\pi_{1} \quad$ defined by eq B3

$\rho_{\mathrm{i}} \quad$ bulk density of the $i$ th constituent

$\rho_{\mathrm{ij}} \quad \rho_{\mathrm{i}}$ in $R_{\mathrm{j}}$

$\sigma \quad$ effective stress defined by eq $60 \mathrm{~b}$

$\sigma_{1} \quad$ defined by eq $59 \mathrm{a}$

$\phi_{0} \quad$ empirical function of $T$ defined by eq $55 a$

$\phi_{01} \quad$ value of $\phi_{0}$ at $T=T_{1}$

$\phi_{1} \quad$ empirical function of $T$ defined by eq $55 \mathrm{~b}$

$\phi_{11}$ value of $\phi_{1}$ at $T=T_{1}$

$\phi_{2} \quad$ empirical function of $T$ defined by eq $55 \mathrm{c}$

$\phi_{21} \quad$ value of $\phi_{2}$ at $T=T_{1}$

$\psi \quad$ some function of $x$ and $t$

$|\psi|$ jump of $\psi$ defined by eq $11 \mathrm{~b}$

$\psi^{+} \quad$ defined by eq $12 b$

$\psi$ defined by eq 12a

* superscript used to indicate the value of any variable evaluated when a point $\left(\alpha_{1}, \alpha_{0}\right)$ in the diagram of temperature gradients is on $R_{\mathrm{s}}^{*}$ 


\title{
Traveling Wave Solutions to the Problem of Quasi-Steady Freezing of Soils
}

\author{
YOSHISUKE NAKANO
}

\section{INTRODUCTION}

We will consider the one-directional steady growth of an ice layer. Let the freezing process advance from the top down and the coordinate $x$ be positive upward with its origin fixed at some point in the unfrozen part of the soil. A freezing soil in this problem may be considered to consist of three parts: the unfrozen part $R_{0}$, the frozen fringe $R_{1}$ and the ice layer $R_{2}$ as shown in Figure 1. The physical properties of parts $R_{0}$ and $R_{2}$ are well understood, but our knowledge of the physical properties and the dynamic behavior of part $R_{1}$ does not ap pear sufficient for engineering applications.

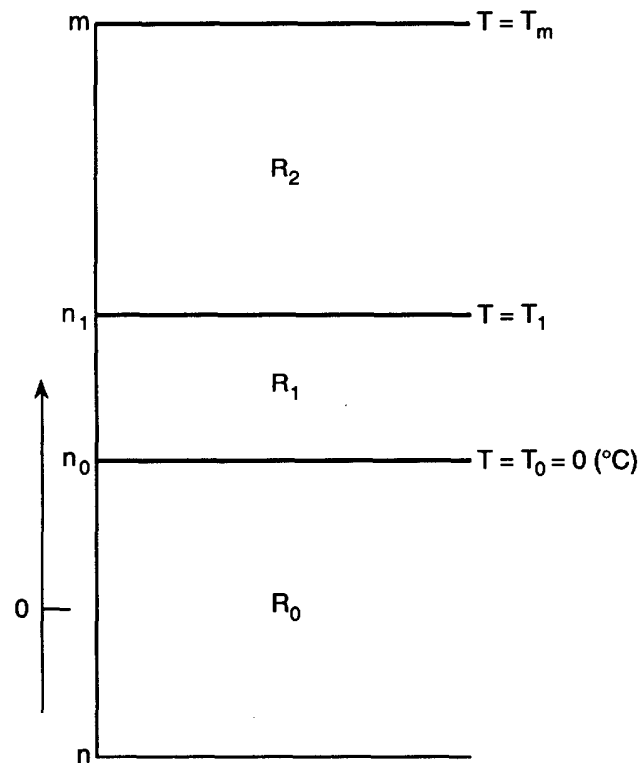

Figure 1. Steady growth of ice-rich frozen soil.
The results of our mathematical and experimental study on the steady growth condition of an ice layer were presented in the three previous reports (Nakano 1990, Takeda and Nakano 1990, Nakano and Takeda 1991). These results clearly show that the model $M_{1}$ accurately describes the properties of a frozen fringe during the steady growth of an ice layer under negligible overburden pressure. The model $M_{1}$ is the frozen fringe where ice may exist but does not grow during the steady growth of an ice layer and the mass flux of water $f_{1}$ is given as

$$
f_{1}=-K_{1} \frac{\partial P_{1}}{\partial x}-K_{2} \frac{\partial T}{\partial x}
$$

where $x$ is the space coordinate, and $K_{1}$ and $K_{2}$ are the properties of a given soil that generally depend on temperature $T$ and the composition of the soil.

Nakano (1990) has shown that the velocity $\dot{n}_{0}\left(=d n_{0} / d t\right)$ of the frost front is nonpositive and vanishes when the steady growth of an ice layer occurs. In this work we will study a case in which $\dot{n}_{0}$ is a given negative constant and the steady growth of ice-rich frozen soil, instead of an ice layer, takes place. In such a case the frozen fringe $R_{1}$ also moves downward with a constant speed. Ice may or may not exist in $R_{1}$. However, if a certain steady distribution of ice is present in $R_{1}$, then the growth of ice must occur in $R_{1}$ because unlike the case of a steadily growing ice layer, the velocity of soil particles in $R_{1}$ relative to $\dot{n}_{0}$ does not vanish when $\dot{n}_{0}<0$. Because of this we must modify the definition of $M_{1}$ so that ice may grow in $R_{1}$ when $\dot{n}_{0}<0$. 
The objective of this work is to show that there exists a traveling wave solution to the problem of steadily growing ice-rich frozen soil and that this solution is reduced to the solution to the problem of a steadily growing ice layer obtained in the previous report (Nakano 1990) when the velocity $\dot{n}_{0}$ vanishes. We will also show that the condition of a the steady growth of ice-rich frozen soils under given hydraulic conditions and applied pressures is uniquely determined by a set of two physical variables, such as $\alpha_{0}$ and $\alpha_{1}$, used in the previous reports.

\section{BASIC EQUATIONS}

We will treat the soil as a mixture of water in the liquid phase $B_{1}$, ice $B_{2}$ and soil minerals $B_{3}$ with bulk densities $\rho_{1}, \rho_{2}$ and $\rho_{3}$, respectively. If $d_{i}$ is the density of the $i$ th constituent, then the volumetric content $\theta_{\mathrm{i}}$ of the $i$ th constituent is given as

$$
\theta_{\mathrm{i}}=\rho_{\mathrm{i}} / d_{\mathrm{i}} .
$$

It is clear that the sum of $\theta_{\mathrm{i}}$ should be unity, namely

$$
\theta_{1}+\theta_{2}+\theta_{3}=1
$$

We will assume that the density of each constituent of the mixture remains constant. Thus, the results of this study are accurate if the deformation of each constituent is negligibly small, regardless of overburden pressure. The dry density of the unfrozen part of the soil is assumed constant during the freezing process.

We will assume that the unfrozen part of the soil is kept saturated with water at all times by using an appropriate device of water supply. The balance of mass for the $i$ th constituent is given (Nakano 1986) as

$$
\frac{\partial}{\partial t} \rho_{\mathrm{i}}=-\frac{\partial}{\partial x}\left(\rho_{\mathrm{i}} v_{\mathrm{i}}\right)+\lambda_{\mathrm{i}}, \quad i=1,2,3
$$

where $v_{\mathrm{i}}$ is the velocity of the $i$ th constituent and $\lambda_{\mathrm{i}}$ is the time rate of supply of mass of the ith constituent per unit volume of the mixture. It should be mentioned that the summation convention on in$\operatorname{dex} i$ is not in force here, so that $\left(\rho_{\mathrm{i}} v_{\mathrm{i}}\right)$ represents only one term. Since none of the constituent is involved in chemical reaction, we have

$$
\lambda_{1}+\lambda_{2}=0 \text { and } \lambda_{3}=0 .
$$

The balance of heat for the $i$ th constituent is given as (Nakano 1986)

$$
\frac{\partial}{\partial t}\left(\rho_{\mathrm{i}} h_{\mathrm{i}}\right)=-\frac{\partial}{\partial x}\left(\rho_{\mathrm{i}} h_{\mathrm{i}} v_{\mathrm{i}}\right)-\frac{\partial}{\partial x} q_{\mathrm{i}}, \quad i=1,2,3
$$

where $\rho_{\mathrm{i}} h_{\mathrm{i}}$ is the heat content of the $i$ th constituent per unit bulk volume and $q_{\mathrm{i}}$ the heat flux by conduction. We assume that the constituents are locally in thermal equilibrium with each other, i.e., that the constituents have locally a common temperature $T\left({ }^{\circ} \mathrm{C}\right)$. Under such an assumption, the heat content $h_{\mathrm{i}}$ is given as

$$
\begin{aligned}
& h_{1}=c_{1}\left(T-T_{0}\right) \\
& h_{2}=-L+c_{2}\left(T-T_{0}\right) \\
& h_{3}=c_{3}\left(T-T_{0}\right) .
\end{aligned}
$$

We sum up eq 6 for $i=1,2,3$ to obtain the heat balance equation of the mixture given as

$$
\frac{\partial}{\partial t} \sum_{i} \rho_{i} h_{i}=-\frac{\partial}{\partial x} \sum_{i} \rho_{i} h_{i} v_{i}-\frac{\partial}{\partial x} q
$$

where $q$ is defined as

$$
q=\sum_{\mathrm{i}} q_{\mathrm{i}}
$$

It is known that $q_{\mathrm{i}}$ depends on the bulk density $\rho_{\mathrm{i}}$, the thermal properties of $i$ th constituent, the temperature gradient and the way in which the $i$ th constituent is distributed in the mixture. For the sake of simplicity we will approximate $q$ as

$$
q=-k \frac{\partial T}{\partial x}
$$

where $k$ is the thermal conductivity of the mixture that generally depends on the thermal properties of each constituent and the composition of the mixture.

Using eq 4 , we reduce eq $8 \mathrm{a}$ to

$$
\sum_{\mathrm{i}} \rho_{\mathrm{i}} \frac{\partial}{\partial t} h_{\mathrm{i}}=-\sum_{\mathrm{i}} h_{\mathrm{i}} \lambda_{\mathrm{i}}-\sum_{\mathrm{i}} \rho_{\mathrm{i}} v_{\mathrm{i}} \frac{\partial}{\partial x} h_{\mathrm{i}}-\frac{\partial}{\partial x} q \cdot(9)
$$

We will assume that $c_{\mathrm{i}}$ and $L$ in eq $7 \mathrm{a}, 7 \mathrm{~b}$ and $7 \mathrm{c}$ do not depend on $T$. Choosing $T_{0}$ to be $0\left({ }^{\circ} \mathrm{C}\right)$ and using eq $7 a, 7 b$ and $7 c$, we reduce eq 9 to:

$$
\frac{\partial}{\partial x} q=L\left(\lambda_{2}+z\right)
$$


where $z$ is defined as

$$
\begin{gathered}
L z=-c \frac{\partial T}{\partial t}+\left(c_{1}-c_{2}\right) T \lambda_{2}-\sum_{\mathrm{i}} \rho_{\mathrm{i}} v_{\mathrm{i}} c_{\mathrm{i}} \frac{\partial T}{\partial t} \\
c=c_{1} \rho_{1}+c_{2} \rho_{2}+c_{3} \rho_{3} .
\end{gathered}
$$

We will now consider a moving surface whose location is given as

$$
x=n(t) \leq m(t) .
$$

In a neighborhood of $n(t)$ we choose two moving surfaces $n^{-}(t)$ and $n^{+}(t)$ with $n^{-}(t)>n(t)>n^{+}(t)$. The jump of a quantity $\psi(x, t)$ at $n(t)$ is defined as

$$
|\psi|=\psi^{-}-\psi^{+}
$$

where

$$
\begin{aligned}
& \psi^{-}=\lim _{n^{-} \rightarrow n} \psi \\
& \psi^{+}=\lim _{n^{+} \rightarrow n} \psi
\end{aligned}
$$

It is clear that $|\psi|=0$ if $\psi$ is continuous at $n(t)$.

Jump conditions at $n(t)$ under the assumption of a continuous $T$ are given (Nakano 1986) as

$$
\begin{aligned}
& \left|\rho_{\mathrm{i}} v_{\mathrm{i}}\right|=\left|\rho_{\mathrm{i}}\right| \dot{n}+\bar{\lambda}_{\mathrm{i}} \quad i=1,2,3 \\
& |q|=\left|\sum_{\mathrm{i}} \rho_{\mathrm{i}} h_{\mathrm{i}}\right| \dot{n}-\left|\sum_{\mathrm{i}} v_{\mathrm{i}} \rho_{\mathrm{i}} h_{\mathrm{i}}\right|
\end{aligned}
$$

where $\bar{\lambda}_{i}$ is the surface supply of the mass of the $i$ th constituent defined as

$$
\bar{\lambda}_{\mathrm{i}}=\lim _{n^{+}, n^{-} \rightarrow n} \int_{n^{+}}^{n^{-}} \lambda_{\mathrm{i}} d x
$$

and $\dot{n}$ is assumed to be continuous and is defined as

$$
\dot{n}=\frac{d n}{d t}
$$

From eq 5 we obtain

$$
\bar{\lambda}_{1}+\bar{\lambda}_{2}=0 \quad \text { and } \quad \bar{\lambda}_{3}=0 .
$$

Thejump conditions, eq $13 \mathrm{a}$ and $13 \mathrm{~b}$, arenecessary and sufficent conditions for the conservation law of heat and mass to hold at $n$. In other words, the conservation law of either heat or mass must be violated if one of these conditions does not hold true at $n$.

\section{Quasi-steady problem}

We will consider a special case in which a frost front $x=n_{0}(t)$ moves with a constant velocity $\dot{n}_{0}$. In such a case we will seek a quasi-steady solution to the problem described by eq 4 and 10a in the form of a traveling wave. We will introduce a new independent variable $\xi$ defined as

$$
\xi=x-\dot{n}_{0} t
$$

where $\dot{n}_{0}=d \dot{n}_{0}(t) / d t$. Using eq 16 we reduce eq 4 to

$$
\frac{d}{d \xi} \rho_{\mathrm{i}}\left(v_{\mathrm{i}}-\dot{n}_{0}\right)=\lambda_{\mathrm{i}}, \quad i=1,2,3
$$

For the sake of convenience we will define new dependent variables $f_{1}$ and $f_{2}$ as

$$
\begin{aligned}
& f_{1}=\rho_{1}\left(v_{1}-v_{3}\right) \\
& f_{2}=\rho_{2}\left(v_{2}-v_{3}\right)
\end{aligned}
$$

It is easy to see that $f_{\mathrm{i}}(i=1,2)$ is the mass flux of either $B_{1}$ or $B_{2}$ relative to the mass flux of soil particles. Using eq $18 \mathrm{a}$ and $18 \mathrm{~b}$, we reduce eq 17 to

$$
\begin{aligned}
& \left(\rho_{1} V\right)^{\prime}=-f_{1}^{\prime}-\lambda_{2} \\
& \left(\rho_{2} V\right)^{\prime}=-f_{2}^{\prime}+\lambda_{2} \\
& \left(\rho_{3} V\right)^{\prime}=0
\end{aligned}
$$

where primes denote differentiation with respect to $\xi$ and $V$ is defined as

$$
V=v_{3}-\dot{n}_{o} .
$$

Using eq $16,18 a$ and $18 b$, we will reduce eq $10 a$ and $10 \mathrm{~b}$ to

$$
\begin{aligned}
& q^{\prime}=-\left(k T^{\prime}\right)^{\prime}=L\left(\lambda_{2}+z\right) \\
& L z=-\left(c_{1} f_{1}+c_{2} f_{2}+c V\right) T^{\prime}+\left(c_{1}-c_{2}\right) \lambda_{2} T .
\end{aligned}
$$

\section{Traveling wave solution}

We will derive a traveling wave solution that satisfies the jump conditions, eq $13 \mathrm{a}$ and $13 \mathrm{~b}$, at $n_{1}$, and the balance equations of mass and heat, eq 19a $19 \mathrm{~b} \mathrm{19} \mathrm{c}$ and 21a. We will assume that the boundaries $n, n_{0}, n_{1}$ and $m$ in Figure 1 move with the same 
constant velocity, namely:

$$
\dot{n}=\dot{n}_{0}=\dot{n}_{1}=\dot{m} .
$$

The pressure $P_{1}$ of water is assumed to be a given constant at $n$ as

$$
P_{1}(n)=P_{1_{n}} .
$$

According to $M_{1}$ (Nakano 1990), the mass flux of water $f_{11}$ in $R_{1}$ is given as

$$
\begin{aligned}
& f_{11}=-K_{1} \frac{\partial P_{1}}{\partial \xi}-K_{2} \frac{\partial T}{\partial \xi} \quad \text { for } \xi \text { in } R_{1} \\
& K_{2} / K_{1} \rightarrow \gamma \quad \text { as } \quad f_{11} \rightarrow 0 \\
& \lim _{\substack{\xi \rightarrow n_{1} \\
\xi}} P_{1}(\xi)=P_{2}\left(n_{1}\right)=P_{21} \\
& R_{1}
\end{aligned}
$$

where $P_{2}$ is the pressure of the frozen part of the soil $R_{2}$ and $\gamma$ is a constant with the value of 1.12 $\mathrm{MPa}{ }^{\circ} \mathrm{C}^{-1}$.

We will also assume that the composition of the soil is continuous and $\bar{\lambda}_{2}$ vanishes at $n_{0}$ as

$$
\left|\rho_{i}\right|=0, \quad \bar{\lambda}_{2}=0 \quad \text { at } n_{0} .
$$

The assumption of eq $22 \mathrm{f}$ implies that the velocity $v_{\mathrm{i}}$ and the flux of heat $\mathrm{q}$ are continuous at $n_{0}$. We will assume that the movement of ice relative to soil particles is negligibly small everywhere, that is

$$
f_{2}=0 \quad \text { in } R_{\mathrm{i}}(i=0,1,2) .
$$

As we discussed (Nakano 1990), when the steady growth of an ice layer occurs, the pressure $P_{1}$ is continuous but the first derivative $d P_{1} / d x$ of $P_{1}$ may be discontinuous at $n_{0}$. Therefore, the boundary $n_{0}$ is generally a free boundary where the first derivative $d P_{1} / d \xi$ may be discontinuous. Finally we will assume that $\rho_{1}$ is given as

$$
\rho_{1}=\rho_{3} v(T)
$$

where $v(T)$ is a given empirically determined function of $T$ that is assumed to be approximated by the equilibrium unfrozen water content at $T$.

We will now denote the values of $v_{\mathrm{i}}$ and $V$, for instance, in the part $R_{\mathrm{j}}(j=0,1,2)$ by $v_{\mathrm{ij}}$ and $V_{\mathrm{j}}$, respectively. We will denote the limiting value of $V_{1}$, for instance, as $\xi$ approaches $n_{1}$ with $\xi<n_{1}$ by
$V_{1}^{+}$and that of $V_{1}$ as $\xi$ approaches $n_{0}$ with $\xi>n_{0}$ by $V_{1}^{-}$as shown in Figure 2. Our immediate task is to reduce the number of unknown variables appearing in this figure by using the jump conditions and the balance equations of mass and heat.

Integrating eq $19 \mathrm{a}, 19 \mathrm{~b}$ and $19 \mathrm{c}$ from $\xi=n_{\overline{1}}$ to $\mathrm{m}^{+}$, we obtain

$$
\begin{aligned}
& f_{12}^{+}+\rho_{12}^{+} V_{2}^{+}=f_{12}^{-}+\rho_{12}^{-} V_{2}^{-}-\Lambda_{1}^{+} \\
& \rho_{22}^{+} V_{2}^{+}=\rho_{22}^{-} V_{2}^{-}+\Lambda_{1}^{+} \\
& \rho_{32}^{+} V_{2}^{+}=\rho_{32}^{-} V_{2}^{-}
\end{aligned}
$$

where

$$
\begin{aligned}
& V_{2}^{+}=v_{32}^{+}-\dot{n}_{0}=r-\dot{n}_{0} \\
& V_{2}^{-}=v_{32}^{-}-\dot{n}_{0} \\
& \Lambda_{1}^{+}=\int_{n_{\overline{1}}}^{m^{+}} \lambda_{2} d \xi
\end{aligned}
$$

where it is assumed that the temperature $T_{\mathrm{m}}$ at $\xi=$ $m$ is low so that $f_{12}^{+}$and $\rho_{12}^{+}$vanish. This implies that $v_{32}^{+}$is equal to the rate of frost heave $r$. Eliminating $\Lambda \neq$, we will reduce eq $23 a, 23 b$ and $23 c$ to

m

$$
v_{2}^{+}, \quad \rho_{12}^{+}, \quad \rho_{22}^{+}, \quad \rho_{32}^{+}, f_{12}^{+}
$$

$R_{2}$

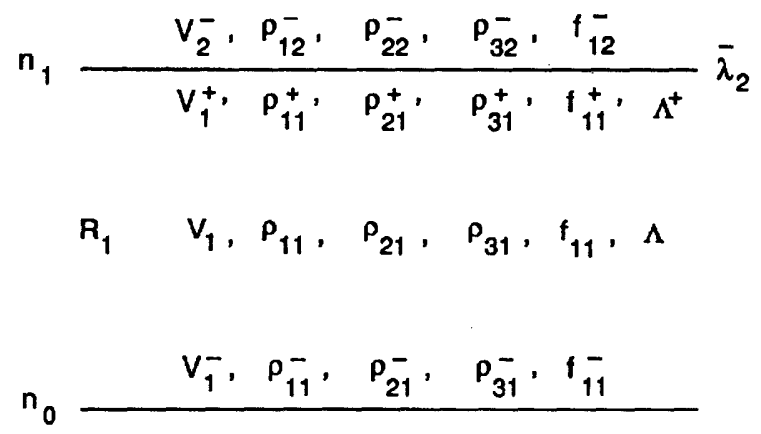

$R_{0} \quad V_{0}, \rho_{10}, \rho_{30}, f_{10}$

Figure 2. Variables in $\mathrm{R}_{0}, \mathrm{R}_{1}$ and $\mathrm{R}_{2}$. 


$$
\begin{aligned}
& \rho_{22}^{+} V_{2}^{+}=\left(v_{1} \rho_{32}^{-}+\rho_{22}^{-}\right) V_{2}^{-}+f_{12}^{-} \\
& \rho_{32}^{+} V_{2}^{+}=\rho_{32}^{-} V_{2}^{-}
\end{aligned}
$$

where $v_{1}$ is defined as:

$$
v_{1}=v\left(T_{1}\right) \text {. }
$$

Using eq 3 , we obtain

$$
\begin{aligned}
& \rho_{22}^{+}=d_{2}\left(1-d_{3}^{-1} \rho_{32}^{+}\right) \\
& \rho_{22}^{-}=d_{2}\left[1-\left(v_{1} d_{1}^{-1}+d_{3}^{-1}\right) \rho_{32}^{-}\right] .
\end{aligned}
$$

From eq 23f, 23i and 23j, we obtain

$$
V_{2}^{+}=\left[1+v_{1}\left(d_{2}^{-1}-d_{1}^{-1}\right) \rho_{32}^{-}\right] V_{2}^{-}+d_{2}^{-1} f_{12}^{-} \text {. }
$$

It is easy to see that all variables at $\mathrm{m}^{+}$are determined if all variables at $n_{1}^{-}$are known. Using eq 13a at $n_{1}$, we obtain

$$
\begin{aligned}
& f_{11}^{+}+\rho_{11}^{+} V_{1}^{+}=f_{12}+\rho_{12}^{-} V_{2}^{-}+\bar{\lambda}_{2} \\
& \rho_{21}^{+} V_{1}^{+}=\rho_{22}^{-} V_{2}^{-}-\overline{\lambda_{2}} \\
& \rho_{31}^{+} V_{1}^{+}=\rho_{32}^{-} V_{2}^{-}
\end{aligned}
$$

where

$$
V_{1}^{+}=v_{31}^{+}-\dot{n}_{0} .
$$

For the sake of simplicity, we will introduce a new variable $\mu$ defined as

$$
\rho_{32}^{-}=\mu \rho_{31}^{+} \text {. }
$$

Using eq $22 \mathrm{~h}$ and $24 \mathrm{e}$, we will reduce eq $24 \mathrm{a}, 24 \mathrm{~b}$ and $24 \mathrm{c}$ to

$$
\begin{aligned}
& f_{11}^{+}+v_{1} \rho_{31}^{+} V_{1}^{+}=f_{12}^{-}+v_{1} \mu \rho_{31}^{+} V_{2}^{-}+\bar{\lambda}_{2} \\
& \rho_{21}^{+} V_{1}^{+}=\rho_{22}^{-} V_{2}^{-}-\bar{\lambda}_{2} \\
& V_{1}^{+}=\mu V_{2}^{-} .
\end{aligned}
$$

Using eq 3, we obtain

$$
\begin{aligned}
& v_{1} \mu \rho_{31}^{+} d_{1}^{-1}+\rho_{22}^{-} d_{2}^{-1}+\mu \rho_{31}^{+} d_{3}^{-1}=1 \\
& v_{1} \rho_{31}^{+} d_{1}^{-1}+\rho_{21}^{+} d_{2}^{-1}+\rho_{31}^{+} d_{3}^{-1}=1
\end{aligned}
$$

From eq 13b, we obtain

$$
|q|=\bar{\lambda}_{2}\left[L+\left(c_{1}-c_{2}\right) T_{1}\right]
$$

Integrating eq $19 \mathrm{a} 19 \mathrm{~b}$ and $19 \mathrm{c}$ from $\xi=n_{0}$ to $\xi$, we obtain the following equations given as

$$
\begin{aligned}
& f_{11}+v \rho_{31} V_{1}=f_{10}+\rho_{10} V_{1}^{-}-\Lambda \\
& \rho_{21} V_{1}=\Lambda \\
& \rho_{31} V_{1}=\rho_{30} V_{1}^{-}
\end{aligned}
$$

where

$$
\begin{aligned}
& V_{1}=V_{31}-\dot{n}_{0} \\
& V_{1}^{-}=V_{0}=-\dot{n}_{0} \\
& \Lambda(\xi)=\int_{n_{0}}^{\xi} \lambda_{2} d \xi, \quad \xi \geq n_{0} .
\end{aligned}
$$

Using eq 3 , we obtain

$$
\begin{aligned}
& v \rho_{31} d_{1}^{-1}+\rho_{21} d_{2}^{-1}+\rho_{31} d_{3}^{-1}=1 \\
& \rho_{10} d_{1}^{-1}+\rho_{30} d_{3}^{-1}=1
\end{aligned}
$$

Taking limits of eq $26 \mathrm{a}, 26 \mathrm{~b}$ and $26 \mathrm{c}$ as $\xi$ approaches $n_{1}$, we obtain

$$
\begin{aligned}
& f_{11}^{+}+v_{1} \rho_{31}^{+} V_{1}^{+}+\Lambda^{+}=f_{10}+\rho_{10} V_{0} \\
& \rho_{21}^{+} V_{1}^{+}-\Lambda^{+}=0 \\
& \rho_{31}^{+} V_{1}^{+}=\rho_{30} V_{0} .
\end{aligned}
$$

Comparing eq $25 \mathrm{a}, 25 \mathrm{~b}$ and $25 \mathrm{c}$ with eq $27 \mathrm{a}, 27 \mathrm{~b}$ and $27 \mathrm{c}$, respectively, we obtain

$$
\begin{aligned}
& f_{12}^{-}+\nu_{1} \mu \rho_{31}^{+} V_{2}^{-}=f_{10}+\rho_{10} V_{0}-\Lambda^{+}-\bar{\lambda}_{2} \\
& \rho_{22}^{-} V_{2}^{-}=\Lambda^{+}+\bar{\lambda}_{2} \\
& \mu V_{2}^{-}=\left(\rho_{30} / \rho_{31}^{+}\right) V_{0} .
\end{aligned}
$$

Let us assume for the time being that $\dot{n}_{0}, v_{1}$ (or $T_{1}$ ) and $\lambda_{2}$ are given. It is easy to see that the left-hand terms of eq 28a, 28b and 28c contain four unknown variables at $n_{1}^{-}$in $R_{2} ; V_{2}^{-}, \rho_{22}^{-}, \mu$, and $f_{12}^{-}$, while the right-hand terms of these three equations contain unknown variables in the combined region of $R_{0}+$ $R_{1}$. Since $\rho_{22}^{-}$and $\mu$ are related by eq $25 \mathrm{~d}$, all the unknown variables at $n_{1}^{-}$listed in Figure 2 are uniquely determined if all the variables in $R_{0}+R_{1}$ listed in Figure 2 are known. 
From eq 27a, 27b and 27c we find that these three equations contain five unknown limiting values, $V_{1}^{+}, \rho_{21}^{+}, \rho_{31}^{+}, f_{11}^{+}$and $\Lambda^{+}$. Since $\rho_{21}^{+}$and $\rho_{31}^{+}$ are related by eq $25 \mathrm{e}$, we have actually four unknown limiting values and three equations. Therefore, if one of these four unknowns is given, then all six unknown limiting values at $n_{1}^{+}$listed in Figure 2 are uniquely determined. Choosing $V_{1}$ to be an independent variable, we will write all other limiting values as follows. First from eq $27 c$, we obtain:

$$
\begin{aligned}
& \rho_{31}^{+}=\rho_{30}\left(V_{0} / V_{1}^{+}\right) \\
& \rho_{11}^{+}=v_{1} \rho_{30}\left(V_{0} / V_{1}^{+}\right) .
\end{aligned}
$$

From eq 25e, we obtain:

$$
\rho_{21}^{+}=d_{2}-d_{2}\left(v_{1} d_{1}^{-1}+d_{3}^{-1}\right) \rho_{30}\left(V_{0} / V_{1}^{+}\right) .
$$

From eq $27 \mathrm{a}$ and $27 \mathrm{~b}$, we obtain

$$
\begin{aligned}
& f_{11}^{+}=f_{10}+\rho_{10} V_{0}-\left(v_{1} \rho_{31}^{+}+\rho_{21}^{+}\right) V_{1}^{+} \\
& \Lambda^{+}=\rho_{21}^{+} V_{1}^{+} .
\end{aligned}
$$

Substituting $\rho_{21}^{+}$in eq 30 a by eq $29 c$, we will reduce eq 30 a to

$$
f_{11}^{+}=f_{10}+s^{+} V_{0}-d_{2}\left(V_{1}^{+}-V_{0}\right)
$$

where $s^{+}$is defined as

$$
s^{+}=\left(1-d_{1}^{-1} d_{2}\right)\left(\rho_{10}-v_{1} \rho_{30}\right) .
$$

Unknown variables, $\rho_{31}, \rho_{11}, \rho_{21}, f_{11}$ and $\Lambda$ are given by eq 29a, 29b, 29c, 31 a and $30 b$, respectively, in which superscripts + are deleted and $v_{1}$ is replaced by $v$. For instance, $f_{11}$ is given as

$$
f_{11}=f_{10}+s V_{0}-d_{2}\left(V_{1}-V_{0}\right)
$$

where $s$ is given as

$$
s=\left(1-d_{1}^{-1} d_{2}\right)\left(\rho_{10}-v \rho_{30}\right) .
$$

Using eq $28 \mathrm{a}$ and $28 \mathrm{c}$, we will write unknown variables at $n_{1}^{-}$as

$$
\begin{aligned}
& V_{2}^{-}=d_{2}^{-1} \bar{\lambda}_{2}+V_{1}^{+} \\
& \mu=V_{1}^{+}\left(V_{1}^{+}+d_{2}^{-1} \bar{\lambda}_{2}\right)^{-1} \\
& \rho_{32}^{-}=\mu \rho_{31}^{+}
\end{aligned}
$$

$$
\begin{aligned}
& \rho_{12}^{-}=v_{1} \mu \rho_{31}^{+} \\
& \rho_{22}^{-}=d_{1}\left[1-\left(v_{1} d_{1}^{-1}+d_{3}^{-1}\right) \mu \rho_{31}^{+}\right] \\
& f_{12}^{-}=f_{11}^{+}-\bar{\lambda}_{2} .
\end{aligned}
$$

Using eq $31 \mathrm{a}$, we will reduce eq $33 \mathrm{f}$ to

$$
f_{12}^{-}=f_{10}+s^{+} V_{0}-d_{2}\left(V_{1}^{+}-V_{0}\right)-\bar{\lambda}_{2} .
$$

In actual experiments, $\rho_{10}$ and $\rho_{30}$ are given as initial conditions. If $V_{0}, v_{1}$ (or $\left.T_{1}\right), \lambda_{2}, f_{10}$ and $V_{1}$ are given, then all other variables are uniquely determined. Since $\rho_{31}^{+}$is difficult to measure experimentally, it is convenient to introduce a new variable $\mu_{0}$ defined as

$$
\mu_{0}=\rho_{32}^{+} \rho_{30}^{-1}=\left(\rho_{32}^{+} / \rho_{32}^{-}\right)\left(\rho_{31}^{+} / \rho_{30}\right) \mu .
$$

Using eq $23 \mathrm{~h}, 25 \mathrm{c}$ and $27 \mathrm{c}$, we will reduce eq $34 \mathrm{a}$ to

$$
\mu_{0}=V_{0} / V_{2}^{+} .
$$

Using eq $24 \mathrm{e}$ and $25 \mathrm{c}$, we will reduce eq $23 \ell$ to

$V_{2}^{+}=\mu^{-1}\left[1+v_{1}\left(d_{1}^{-1}-d_{2}^{-1}\right) \mu p_{31}^{+}\right] V_{1}^{+}+d_{2}^{-1} f_{12}^{-}$

Substituting $f_{12}^{-}$in eq $34 \mathrm{c}$ by eq $33 \mathrm{~g}$ and using eq $33 b$, we will reduce eq $34 c$ to

$$
V_{2}^{+}=d_{2}^{-1} f_{10}+V_{0}\left[1+\left(d_{2}^{-1}-d_{1}^{-1}\right) \rho_{10}\right] .
$$

Combining eq $34 \mathrm{~b}$ and $34 \mathrm{~d}$, we obtain

$$
\mu_{0}=V_{0}\left\{d_{2}^{-1} f_{10}+V_{0}\left[1+\left(d_{2}^{-1}-d_{1}^{-1}\right)\right] \rho_{10}\right\}^{-1} \text {. }
$$

From eq $23 \mathrm{~d}$ and $34 \mathrm{~d}$ we obtain

$$
r=d_{2}^{-1} f_{10}+\left(d_{2}^{-1}-d_{1}^{-1}\right) \rho_{10} V_{0}
$$

Using eq $34 f$, we will reduce eq $34 \mathrm{e}$ to

$$
\mu_{0}=V_{0}\left(r+V_{0}\right)^{-1} \text {. }
$$

We will introduce new variables, $w_{\mathrm{ij}}$ and $w_{\mathrm{j}}$, defined as

$$
\begin{aligned}
& w_{\mathrm{ij}}=\rho_{\mathrm{ij}} \rho_{3 \mathrm{j}}^{-1}, \quad i=1,2 \text { and } j=0,1,2 \\
& w_{\mathrm{j}}=w_{1 \mathrm{j}}+w_{\mathrm{j}} \quad j=0,1,2 .
\end{aligned}
$$

It is clear that $w_{\mathrm{ij}}$ is the content of the ith constituent in $R_{\mathrm{j}}$ and that $w_{\mathrm{j}}$ is the content of ice and unfrozen water in $R_{\mathrm{j}}$. We will refer to $w_{\mathrm{j}}$ as the total water 
content. Using $w_{\mathrm{j}}$, we will reduce eq 32a to

$$
f_{11}=f_{10}+\rho_{30}\left(w_{0}-w_{1}\right) V_{0} .
$$

Using eq $26 a, 26 b$ and $36 a$, we obtain

$$
\Lambda=\rho_{30}\left(w_{1}-v\right) V_{0} .
$$

We will now examine the behavior of $f_{11}$. From eq 32a we obtain

$$
f_{11}=f_{10}+s V_{0}-d_{2} v_{31} .
$$

Using eq 26c, we will write $v_{31}$ as:

$$
v_{31}=\left(\rho_{30} \rho_{31}-1\right) V_{0} \text {. }
$$

Since $T$ decreases as $\xi$ increases from $n_{0}$ to $n_{1}, s$ is positive in $R_{1}$ and increases with $\xi$. It is anticipated that $\rho_{31}$ may decrease with $\xi$ but does not increase with $\xi$. Since $V_{0} \geq 0$, from eq $37 \mathrm{~b}$ we find that $v_{31} \geq$ 0 and $v_{31}^{\prime} \geq 0$ in $R_{1}$. Therefore, from eq 37 a we obtain

$$
f_{10}+s^{+} V_{0} \geq f_{11} \geq f_{10}-d_{2} v_{31}^{+}
$$

where

$$
v_{31}^{+}=\left(\rho_{30}-\rho_{31}^{+}\right) V_{0} / \rho_{31}^{+} .
$$

\section{Ice-rich frozen soil}

We will focus the remainder of our analysis on a special case in which the frozen part of the soil contains a significant amount of ice. For such a case the mobility of water in $R_{2}$ is anticipated to be much less than that in $R_{1}$ and we may neglect $f_{12}^{-}$. It follows from eq $34 \mathrm{a}, 34 \mathrm{~g}$ and $36 \mathrm{~b}$ that the values of $\mu_{0}, V_{0}$ and $\Lambda$ remain small.

The exact composition of $R_{1}$ is not known. However, it is a generally accepted view that $\rho_{31}$ does not change significantly from $\rho_{30}$. The results of our analysis on the data of Tomakomai silt (Nakano and Takeda 1991) appear to support such a viewpoint. Assuming the existence of a certain rule for $\rho_{31}$, we will explore probable rules below. Suppose that such a rule is known, then two of five independent variables, $V_{1}$ and $\bar{\lambda}_{2}$, are uniquely determined by eq $26 \mathrm{c}$ and $33 \mathrm{~g}$, respectively, when three remaining independent variables, $V_{0}, T_{1}$ and $f_{10}$, are given in this case. Let us consider first a special rule that $\rho_{31}$ is kept constant at $\rho_{30}$. In such a case $v_{31}$ vanishes and eq $37 \mathrm{a}$ is reduced to

$$
f_{11}=f_{10}+s V_{0} .
$$

When $V_{0}>0$, we obtain

$f_{11}>f_{10}$ and $f_{11}^{\prime}>0$, if $V_{0}>0$ and $\xi>n_{0}$.

It follows from eq $39 \mathrm{~b}$ that $f_{11}$ is greater than $f_{10}$ and increases with $\xi$. This special case does not appear to beprobablebecause the mobility of water should not increase with increasing $\xi$.

Next we will consider a special rule that the total water content $w_{1}$ is kept constant at $w_{0}$. From eq $36 \mathrm{a}$ and $33 \mathrm{f}$ we obtain:

$$
f_{11}=f_{10}=\bar{\lambda}_{2} .
$$

From eq 37a we obtain

$$
v_{31}=d_{2}^{-1} s V_{0} .
$$

From eq 29a we obtain

$$
\rho_{31}=\rho_{30}\left(1+d_{2}^{-1} s\right)^{-1} .
$$

We will reduce eq $36 \mathrm{~b}$ to

$$
\Lambda=\rho_{30}\left(w_{0}-v\right) V_{0} .
$$

It follows from eq $40 \mathrm{~b}$ and $40 \mathrm{c}$ that $v_{31}$ increases with $\xi$ while $\rho_{31}$ decreases with $\xi$ for a given $V_{0}$. This second special case appears more probable than the first case because the mass flux of water should not increase with increasing $\xi$. We will study the second case below. The empirical function $v(T)$ in eq $40 \mathrm{~d}$ is known to be an increasing function of $T$ with $v(0)=w_{0}$. We will assume that $v(T)$ possesses a continuous first derivative.

The thermal conductivity $k_{1}$ of $R_{1}$ depends on the composition of $R_{1}$. Our experimental data indicate that $k_{1}$ is a nondecreasing function of $\xi$. We will approximate $k_{1}$ by a linear function of $\xi$ as

$$
\begin{aligned}
& k_{1}(\xi)=k_{0}\left[1+\eta\left(\xi-n_{0}\right)\right], \quad n_{1}>\xi \geq n_{0} \\
& \eta=\left(k_{11}-k_{0}\right) /\left(\delta k_{0}\right) \geq 0 \\
& \delta=n_{1}-n_{0} \\
& \lim _{\substack{\xi \rightarrow n_{1} \\
\xi \text { in } R_{1}}} k_{1}(\xi)=k_{11} \leq k_{21}
\end{aligned}
$$

where $k_{21}$ is the limiting value of $k_{2}$ when $\xi$ approaches $n_{1}$ while $\xi$ is in $R_{2}$. Under assumptions described above we will study thermal and hydraulic fields below. 


\section{Temperature $T(\xi)$}

We will seek solutions $T(\xi)$ to the balance equation of heat given by eq 21a in $R_{0}$ and $R_{1}$. We will begin with $R_{0}$. Since $f_{1}=f_{10}, f_{2}=0, V=V_{0}$ and $\lambda_{2}=$ 0 , from eq 21a and $21 \mathrm{~b}$ we obtain

$$
\begin{aligned}
& \mathrm{T}^{\prime \prime}-\beta_{0} T^{\prime}=0, \quad \xi \text { in } R_{0} \\
& \beta_{0}=\left(c_{1} f_{10}+c_{0} V_{0}\right) / k_{0}
\end{aligned}
$$

where $k_{0}$ is the thermal conductivity of $R_{0}$ and $c_{0}$ is defined as

$$
c_{0}=c_{1} \rho_{10}+c_{3} \rho_{30} .
$$

Since $T\left(n_{0}\right)=0^{\circ} \mathrm{C}$, integrating eq $41 \mathrm{a}$, we obtain

$$
\begin{aligned}
& T(\xi)=\alpha_{0} \beta_{0}^{-1}\left\{1-\exp \left[-\beta_{0}\left(n_{0}-\xi\right)\right]\right\} \\
& T^{\prime}(\xi)=-\alpha_{0} \exp \left[-\beta_{0}\left(n_{0}-\xi\right)\right]
\end{aligned}
$$

where $\alpha_{0}$ is defined as

$$
\mathrm{a}_{0}=-T^{\prime}\left(n_{0}\right) .
$$

Next we will seek a solution in $R_{1}$. First we will rewrite eq 21a and $21 \mathrm{~b}$ by using eq $26 \mathrm{a}, 26 \mathrm{~b}$ and 26c. Since $f_{1}=f_{10}, f_{2}=0$ and $\lambda_{2}=\Lambda^{\prime}$ in this case, we will reduce eq $21 \mathrm{~b}$ to

$$
L z=-\left(c_{1} f_{10}+c V_{1}\right) T^{\prime}+\left(c_{1}-c_{2}\right) \Lambda^{\prime} T .
$$

Using eq 26a, 26b and 26c, we obtain

$$
\mathrm{c} V_{1}=-\left(c_{1}-c_{2}\right) \Lambda+c_{0} V_{0} \text {. }
$$

Using eq $43 b$, we will reduce eq $43 a$ to

$$
L z=-\left(c_{1} f_{10}+c_{0} V_{0}\right) T^{\prime}+\left(c_{1}-c_{2}\right)(\Lambda T)^{\prime} .
$$

Using eq $43 c$, we will reduce eq $21 a$ to

$$
\mathrm{q}^{\prime}=-k_{0} \beta_{0} T^{\prime}+\left(c_{1}-c_{2}\right)(\Lambda T)^{\prime}+L \Lambda^{\prime} .
$$

Integrating eq $44 \mathrm{a}$ from $\xi=n_{0}$ to $\xi$, we obtain

$$
\begin{aligned}
& q=-k_{1} T^{\prime}=k_{0} \alpha_{0}-z_{1} T+\mathrm{L} \Lambda \\
& z_{1}=k_{0} \beta_{0}-\left(c_{1}-c_{2}\right) \Lambda
\end{aligned}
$$

where $k_{1}$ is the thermal conductivity of $R_{1}$ and $\Lambda$ is given as

$$
\Lambda=\left(w_{0}-v\right) \rho_{30} V_{0} .
$$

Using eq $41 b$ and $44 d$, we will reduce eq $44 c$ to

$$
z_{1}=c_{1} f_{10}+\rho_{30} V_{0}\left[c_{3}+c_{2} w_{0}+\left(c_{1}-c_{2}\right) v\right] \text {. }
$$

Neglecting the last term in eq $44 \mathrm{e}$, we will reduce eq $44 \mathrm{~b}$ to

$$
-k_{1} T^{\prime}=k_{0} \alpha_{0}-k_{0} \beta_{1} T+L \Lambda
$$

where $\beta_{1}$ is defined as

$$
\beta_{1}=k_{0}^{-1}\left[c_{1} f_{10}+\rho_{30} V_{0}\left(c_{3}+c_{2} w_{0}\right)\right]
$$

We will reduce the jump condition (eq 25f) to a somewhat more convenient form by using eq $44 \mathrm{~b}$. We will write the limiting value $q^{-}$when $\xi$ approaches $n_{1}$ while $\xi$ is in $R_{2}$ as

$$
\begin{aligned}
& \mathrm{q}^{-}=k_{21} \alpha_{1} \\
& k_{21}=\lim _{\substack{\xi \rightarrow n_{1} \\
\xi}} k_{2}(\xi)
\end{aligned}
$$

where $k_{2}$ is the thermal conductivity of $R_{2}$ and $\alpha_{1}$ is the absolute value of the limiting temperature gradient as $\xi$ approaches $n_{1}$ while $\xi$ is in $R_{2}$. Using eq $44 \mathrm{~b}$ and 47 , we will reduce eq $25 \mathrm{f}$ to

$$
k_{21} \alpha_{1}-k_{0} \alpha_{0}+k_{0} \beta_{0} T_{1}=\left(f_{10}+\Lambda^{+}\right)\left[L+\left(c_{1}-c_{2}\right) T_{1}\right]
$$

$$
\Lambda^{+}=\left(w_{0}-v_{1}\right) \rho_{30} V_{0} .
$$

As shown in Appendix A, eq 45 has a unique and decreasing solution for $n_{1} \geq \xi \geq n_{0}$. For a special case in which the following condition holds true,

$$
\eta \delta<1 \text { and } \beta_{1} \delta<1 .
$$

We found that the condition, eq 51 , holds true when the steady growth of an ice layer occurs (Takeda and Nakano 1990). When eq51 holds true, we may use an approximation (Nakano 1990) given as

$$
X^{\beta_{1} \eta^{-1}}=1+\beta_{1}\left(\xi-n_{0}\right) .
$$

Weobtain an approximatesolution (App.B) given as

$$
T(\xi)=\pi_{0} \pi_{1}^{-1} \bar{v}-\left(\pi_{1}-\pi_{0} \pi_{1}^{-1} \bar{v} \beta_{1}\right)\left(\xi-n_{0}\right)
$$




$$
\begin{aligned}
T^{\prime}(\xi) X= & -\pi_{1}+\pi_{0}\left(v+\pi_{1}^{-1} \beta_{1} \bar{v}\right) \\
& -\beta_{1}\left(\pi_{1}-\pi_{0} \pi_{1}^{-1} \beta_{1} \bar{v}\right)\left(\xi-n_{0}\right) .
\end{aligned}
$$

It is easy to see from eq 45 that the temperature $T(\xi)$ is uniquely determined if $V_{0}, f_{10}, \alpha_{0}$ and $\xi$ are given. Hence, the temperature $T_{1}$ at $n_{1}$ is determined by $V_{0}, f_{10}, \alpha_{0}$ and $\delta$. Suppose that $V_{0}, f_{10}, \alpha_{0}$ and $\delta$ are given, then $T_{1}$ is determined by eq 45 . Once $T_{1}$ is known, then $\alpha_{1}$ is determined by eq 49. As we described in the preceding section, all variables listed in Figure 2 are determined by $V_{0}, T_{1}$ and $f_{10}$ if $\rho_{31}$ obeys a certain known rule in $R_{1}$. This implies that four independent variables must be given in order to specify the condition of freezing. We will choose $\alpha_{0}, \alpha_{1}, f_{10}$ and $V_{0}$ to be independent variables.

\section{Pressure $\boldsymbol{P}_{\mathbf{1}}(\xi)$}

When the mass flux of water is given by eq 1 , we have found (Nakano 1990) that the following equations hold true in $R_{1}$ :

$$
\begin{gathered}
P_{10}=P_{1_{n}}-\left[\left(f_{10} / K_{0}\right)+p_{0}\right] \delta_{0} \\
P_{21}=P_{10}-\int_{n_{0}}^{n_{1}} K_{1}^{-1} K_{2} T^{v} d \xi-f_{10} \int_{n_{0}}^{n_{1}} K_{1}^{-1} d \xi
\end{gathered}
$$

where $P_{10}=P_{1}\left(n_{0}\right), P_{1_{\mathrm{n}}}=P_{1}(n)$,

$n=$ some point in $R_{0}$

$K_{0}=$ hydraulic conductivity in $R_{0}$

$p_{0}=$ gravity term that is equal to the density $d_{1}$ multiplied by the gravitational acceleration.

$\delta_{0}$ is defined as

$$
\delta_{0}=n_{0}-n>0 .
$$

We will assume that $P_{21}, P_{1_{n}}$ and $\delta_{0}$ are given.

In order to reduce eq $54 \mathrm{~b}$ to a simpler form, we will introduce the following three dimensionless quantities

$$
\phi_{0}(T)= \begin{cases}1 & T_{1}=0 \\ T_{1}^{-1} \int_{0}^{T}\left(K_{10} / K_{1}\right)\left(K_{2} / K_{20}\right) d T & T<0\end{cases}
$$

$\phi_{1}(T)= \begin{cases}1 & T_{1}=0 \\ T_{1}^{-1} \int_{0}^{T}\left(K_{10} / K_{1}\right)\left(k_{1} / k_{0}\right) d T & T<0\end{cases}$

$\phi_{2}(T)=\left\{\begin{array}{lr}1 & T_{1}=0 \\ T_{1}^{-1} \int_{0}^{T}\left(v / w_{0}\right)\left(K_{10} / K_{1}\right)\left(k_{1} / k_{0}\right) d T & T<0\end{array}\right.$

where $K_{10}$ and $K_{20}$ are the limiting values of $K_{1}$ and $K_{2}$, respectively, as $\xi$ approaches $n_{0}$ while $\xi$ in $R_{1}$.

Choosing $T$ as an independent variable, we will write the two integrations in eq $54 \mathrm{~b}$ as

$$
\begin{aligned}
& I_{0} \equiv \int_{n_{0}}^{n_{1}} K_{1}^{-1} K_{2} T^{v} d \xi=K_{10}^{-1} K_{20} T_{1} \phi_{01} \\
& I_{1} \equiv \int_{n_{0}}^{n_{1}} K_{1}^{-1} d \xi=\int_{0}^{T_{1}}\left(K_{1} T^{v}\right)^{-1} d T
\end{aligned}
$$

where $\phi_{01}=\phi_{0}\left(T_{1}\right)$. We will write eq $54 \mathrm{~b}$ as

$$
P_{21}=P_{10}-I_{0}-f_{10} I_{1} \text {. }
$$

Using eq $55 b$ and $55 c$, we will reduce eq $56 b$ to (App.C)

$$
\begin{gathered}
I_{1}=-\left(\pi_{1} K_{10}\right)^{-1}\left[\phi_{11} T_{1}(1-\varepsilon)+w_{0} \pi_{0} \pi_{1}^{-1} \phi_{21} T_{1}\right] \\
\varepsilon=\beta_{1} \pi_{1}^{-1}\left[-T_{1}+\left(\phi_{11}\right)^{-1} \int_{0}^{T_{1}} \phi_{1} d T\right]
\end{gathered}
$$

where $\phi_{11}=\phi_{1}\left(T_{1}\right)$ and $\phi_{21}=\phi_{2}\left(T_{1}\right)$.

Using eq 56a and 58a, we will write eq 57 as

$$
\begin{aligned}
\sigma_{1}= & P_{21}-P_{10}=-T_{1} I_{2} \\
I_{2}= & K_{10}^{-1} K_{20} \phi_{01}-\left(\pi_{1} K_{10}\right)^{-1} \\
& f_{10}\left[\phi_{11}(1-\varepsilon)+w_{0} \pi_{0} \pi_{1}^{-1} \phi_{21}\right] .
\end{aligned}
$$

Using eq $54 a$, we obtain

$$
\begin{aligned}
& \sigma_{1}=\sigma+p_{0} \delta_{0}+\delta_{0} K_{0}^{-1} f_{10} \\
& \sigma=P_{21}-P_{1_{n}}
\end{aligned}
$$




$$
-T_{1}=\left(\sigma+p_{0} \delta_{0}+\delta_{0} K_{0}^{-1} f_{10}\right) / I_{2}
$$

Since the composition of the freezing soil is assumed to be continuous at $n_{0}$, we may expect that the limiting value $K_{10}$ and $K_{0}$ should be equal

$$
K_{0}=K_{10} .
$$

Neglecting the gravity term, we will reduce eq $60 \mathrm{c}$ to

$$
-T_{1}=\left(\sigma+\delta_{0} K_{0}^{-1} f_{10}\right) / I_{2}
$$

When $f_{10}$ vanishes, from eq $61 \mathrm{~b}$ we obtain

$$
\sigma=-\left(K_{20} / K_{0}\right) \phi_{01} T_{1}, \quad \text { if } f_{10}=0 .
$$

The generalized Clausius-Clapeyron equation (Edlefsen and Anderson 1943), which was proven empirically by Radd and Oertle (1973), is given as

$$
\sigma=-\gamma T_{1}, \quad \text { if } f_{10}=0 .
$$

Comparing eq $61 \mathrm{c}$ with eq $62 \mathrm{a}$, we obtain

$$
\gamma=\left(K_{20} / K_{0}\right) \phi_{01}, \quad \text { if } f_{10}=0
$$

It follows from eq $22 \mathrm{~d}$ that eq $62 \mathrm{~b}$ holds true and that we have

$$
\begin{array}{ll}
\gamma=K_{20} / K_{0} & \\
\phi_{01}=1, & \text { if } f_{10}=0 .
\end{array}
$$

It should be noted that eq $62 \mathrm{c}$ should hold true regardless of $f_{10}$. Using eq $62 c$, we reduce eq $59 b$ to

$$
\begin{aligned}
I_{2}= & \gamma \phi_{01}-\left(\pi_{1} K_{0}\right)^{-1} \\
& f_{10}\left[\phi_{11}(1-\varepsilon)+w_{0} \pi_{0} \pi_{1}^{-1} \phi_{21}\right] .
\end{aligned}
$$

For a special case in which $\sigma$ is negligibly small, eq $61 \mathrm{~b}$ is reduced to

$$
-T_{1}=\left(\delta_{0} K_{0}^{-1} f_{10}\right) / I_{2} .
$$

At the end of the preceding section we had four independent variables, $\alpha_{0}, \alpha_{1}, f_{10}$ and $V_{0}$. Since we have derived another equation, eq $60 \mathrm{c}$, we now have three independent variables, $\alpha_{0}, \alpha_{1}$ and $V_{0}$. We will derive one more equation below in order to reduce the number of independent variables to two.

\section{Condition of steady growth}

Since the mass flux is given by eq $22 c$, the flux $f_{10}$ in a neighborhood of $n_{1}$ is given as

$$
f_{10}=-K_{11} P_{1}^{\prime}\left(n_{1}^{+}\right)-K_{21} T^{\prime}\left(n_{1}^{+}\right)
$$

where $K_{11}, P_{1}^{\prime}\left(n_{1}^{+}\right), K_{21}$ and $T^{\prime \prime}\left(n_{1}^{+}\right)$are the limiting values of $K_{1}, P_{1}^{\prime}(\xi), K_{2}$ and $T^{\prime}(\xi)$, respectively, as $\xi$ approaches $n_{1}$ while $\xi$ is in $R_{1}$. From eq $53 \mathrm{~b}$ we obtain

$$
\begin{aligned}
& T^{\prime}\left(n_{1}^{+}\right)=-\alpha_{0} b \\
& b=(1+\eta \delta)^{-1}\left(b_{0}+b_{1} \delta\right) \\
& b=\alpha_{0}^{-1}\left(\pi_{1}-\pi_{0} \nu_{1}-\pi_{0} \pi_{1}^{-1} \beta_{1} \bar{v}_{1}\right) \\
& b_{1}=\alpha_{0}^{-1} \beta_{1}\left(\pi_{1}-\pi_{0} \pi_{1}^{-1} \beta_{1} \bar{v}_{1}\right) .
\end{aligned}
$$

Similarly from eq 53a we obtain

$$
\begin{aligned}
& T_{1}=-\alpha_{0}\left(a_{0}+a_{1} \delta\right) \\
& a_{0}=-\alpha_{0}^{-1} \pi_{0} \pi_{1}^{-1} \bar{v}_{1} \\
& a_{1}=\alpha_{0}^{-1}\left(\pi_{1}-\pi_{0} \pi_{1}^{-1} \bar{v}_{1} \beta_{1}\right)
\end{aligned}
$$

where $\bar{v}_{1}=\bar{v}\left(T_{1}\right)$.

Using eq $63 \mathrm{~b}$, we will reduce eq $63 \mathrm{a}$ to

$$
f_{10}=-K_{11} P_{1}^{\prime}\left(n_{1}^{+}\right)+b K_{21} \alpha_{0} .
$$

Neglecting terms representing sensitive heat, we will reduce eq 49 to

$$
k_{0} \alpha_{0}+L f_{10}=k_{21} \alpha_{1}-\rho_{30} V_{0} L\left(w_{0}-v_{1}\right)
$$

Now we will recall a special case studied (Nakano 1990) where $V_{0}$ vanishes and the steady growth of an ice layer occurs. In such a case, $\pi_{0}$ vanishes and eq $63 \mathrm{c}$ is reduced to

$$
\begin{aligned}
& b=(1+\eta \delta)^{-1}\left(1+\beta_{1} \delta\right) \\
& \beta_{1}=-k_{0}^{-1} c_{1} f_{10} .
\end{aligned}
$$

Also eq $64 \mathrm{~b}$ is reduced to

$$
k_{0} \alpha_{0}+L f_{10}=k_{21} \alpha_{1} \text {. }
$$

It was found (Nakano 1990) that the steady growth of an ice layer occurs under the conditions given as

$$
\left(k_{21} / k_{0}\right) \alpha_{1}>\alpha_{0}
$$




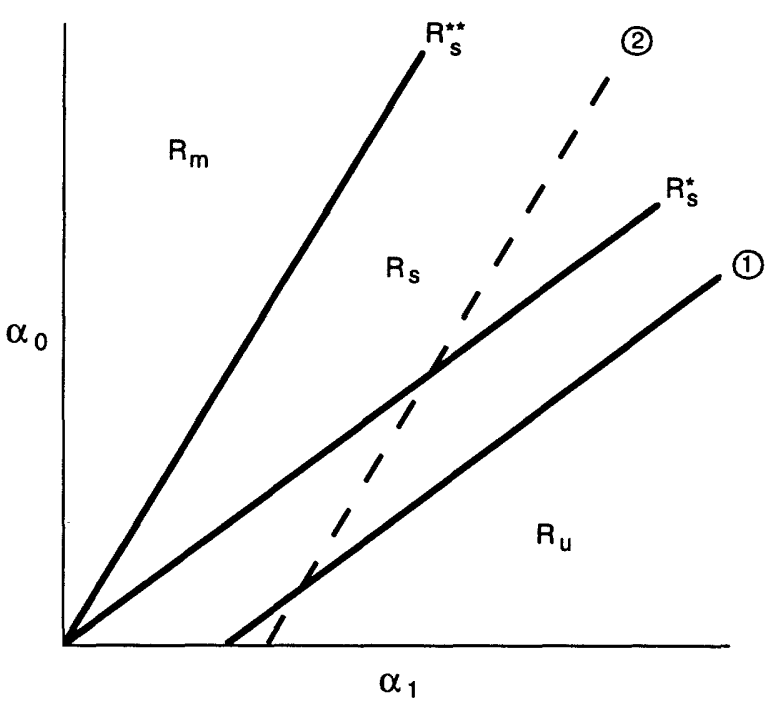

Figure 3. Temperature gradients $\alpha_{1}$ and $\alpha_{0}$.

$$
P_{1}^{\prime}\left(n_{1}^{+}\right)>0 .
$$

Using eq $64 \mathrm{a}$ and $65 \mathrm{c}$ and combining eq $66 \mathrm{a}$ and $66 b$, we obtain

$$
\left(k_{21} / k_{0}\right) \alpha_{1}>\alpha_{0}>k_{21}\left(k_{0}+L b K_{21}\right)^{-1} \alpha_{1} .
$$

The region $R_{\mathrm{s}}$ in Figure 3 satisfies eq $66 \mathrm{c}$ and the steady growth of an ice layer occurs in $R_{\mathrm{s}}$. Line $R_{\mathrm{s}}^{* *}$ in Figure 3 is given as

$$
\alpha_{0}=\left(k_{21} / k_{0}\right) \alpha_{1} .
$$

Line $R_{\mathrm{s}}^{* *}$ is the boundary between $R_{\mathrm{s}}$ and $R_{\mathrm{m}}$ where an ice layer melts. The boundary $R_{\mathrm{s}}^{*}$ in Figure 3 is given as

$$
\alpha_{0}=k_{21}\left(k_{0}+L b^{*} K_{21}^{*}\right)^{-1} \alpha_{1}
$$

where superscripts * are used to indicate the value of any variable when a point $\left(\alpha_{1}, \alpha_{0}\right)$ belongs to $R_{s}^{*}$. Since $b^{*}$ and $K_{21}^{*}$ generally depend on $\alpha_{0}$ and $\alpha_{1}$, the boundary $R_{\mathrm{s}}^{*}$ between $R_{\mathrm{s}}$ and $R_{\mathrm{u}}$, where the steady growth of an ice layer does not occur, is a curve stemming from the origin. From eq $66 \mathrm{~b}$ we obtain

$$
\begin{array}{ll}
P_{1}^{\prime}\left(n_{1}^{+}\right)>0 & \text { in } R_{\mathrm{s}} \\
P_{1}^{\prime}\left(n_{1}^{+}\right)=0 & \text { on } R_{\mathrm{s}}^{*} .
\end{array}
$$

Now we will examine the case in which $V_{0}$ is positive and the steady growth of ice-rich frozen soil occurs. First we will show that the necessary condition for the steady growth is given as

$$
P_{1}^{\prime}\left(n_{1}^{+}\right) \geq 0
$$

Suppose that eq 68 does not hold true. Since $P_{1}^{\prime}(\xi)<0$ in a neighborhood of $n_{1}$ in $R_{1}$, there exists a point $\xi_{1}$ in this neighborhood such that $P_{1}\left(\xi_{1}\right)>P_{21}$. Also $P_{21}>P_{10}$ because $P_{21} \geq 0$ and $f_{10}$ $>0$. Since $P_{1}(\xi)$ is continuous in $R_{1}$, there exists at least one point $\xi_{2}$ with $\xi_{1}>\xi_{2}>n_{0}$ such that $P_{1}\left(\xi_{2}\right)$ $=P_{21}$. This implies that another ice-rich frozen soil layer can exist in $R_{1}$. This is obviously contradictory to our assumption that there is no ice-rich frozen soil layer in $R_{1}$.

When eq 68 holds true, from eq $64 \mathrm{a}$ and $64 \mathrm{~b}$ we obtain

$$
\alpha_{0} \geq k_{21} b_{2}^{-1} \alpha_{1}-b_{3}
$$

where $b_{2}$ and $b_{3}$ are defined as

$$
\begin{aligned}
& b_{2}=k_{0}+L b K_{21} \\
& b_{3}=b_{2}^{-1} \rho_{30} V_{0} L\left(w_{0}-v_{1}\right) .
\end{aligned}
$$

In Figure 3 we will draw curve 1 given as

$$
\alpha_{0}=k_{21} b_{2}^{-1} \alpha_{1}-b_{3} \text {. }
$$

Since $V_{0}>0$, curve 1 must be in $R_{\mathrm{u}}$ and converges to $R_{s}^{*}$ when $V_{0}$ approaches zero. When $V_{0}$ vanishes and the steady growth of an ice layer occurs, a line of constant $f_{10}$ is parallel to $R_{\mathrm{s}}^{* *}$, such as broken line 2 in Figure 3. It follows from eq $64 \mathrm{~b}$ that line 2 is still the line of constant $f_{10}$ except in $R_{\mathbf{u}}$ where line 2 is the line of constant $f_{10}+\rho_{30} V_{0}\left(w_{0}-v_{1}\right)$.

It follows from eq $69 \mathrm{~d}$ that the distance between curve 1 and $R_{s}^{*}$ increases with increasing $V_{0}$. From eq $34 \mathrm{~g}$ we find that the ice content in $R_{2}$ decreases with increasing $V_{0}$. The condition eq 69a implies that the steady growth of frozen soil occurs in the region bounded by curve 1 and $R_{s}^{*}$. Since $V_{0}$ is an arbitrary positive number, eq $69 \mathrm{a}$ also implies that the steady growth of frozen soil occurs everywhere in $R_{\mathrm{u}}$. However, the steady growth of icerich frozen soil is anticipated to occur in the part of $R_{\mathrm{u}}$ not far from the boundary $R_{\mathrm{s}}^{*}$.

Suppose that a point $\left(\alpha_{1}, \alpha_{0}\right)$ in $R_{\mathrm{u}}$ is given; then we can find $V_{0}$ that satisfies eq $69 \mathrm{~d}$. At the end of the preceding section we had three independent variables, $\alpha_{0}, \alpha_{1}$ and $V_{0}$. Since these three variables are related by eq $69 \mathrm{~d}$, we now have only two independent variables, $\alpha_{0}$ and $\alpha_{1}$. In other words, we have found that the condition of the steady growth of ice-rich frozen soil is uniquely determined by two independent variables, $\alpha_{0}$ and $\alpha_{1}$, 
under a given hydraulic condition and overburden pressure. We have shown that there exists a traveling wave solution containing two independent parameters, $\alpha_{0}$ and $\alpha_{1}$, to the problem of steadily growing ice-rich frozen soil.

\section{DISCUSSION}

One of the outstanding questions among researchers of frost heave has been the relationship between the rate of frost heave $r$ and the speed of a frost front $V_{0}$. A significant amount of effort has been made to determine empirically this relationship under the hypothesis that $r$ is uniquely determined by $V_{0}$. The empirical relationships between $r$ and $V_{0}$ reported in the literature sometime disagree with (or even contradict) each other (Takashi et al. 1978), and there appears to be no consensus among researchers. This situation casts a serious doubt upon the validity of the underlying hypothesis that $r$ is uniquely determined by $V_{0}$.

We will show that $r$ is not uniquely determined by $V_{0}$ if $M_{1}$ and the assumptions used in our analysis are valid. Using eq $64 \mathrm{~b}$, we will reduce eq $34 \mathrm{f}$ to

$$
\begin{aligned}
d_{2} r= & L^{-1}\left(k_{21} \alpha_{1}-k_{0} \alpha_{0}\right) \\
& \left.-\rho_{30} V d d_{1}^{-1} d_{2} w_{0}-v_{1}\right) .
\end{aligned}
$$

Since $\alpha_{1}$ and $\alpha_{0}$ are related by eq $69 \mathrm{~d}$, we can express $r$ as a function of $V_{0}$ and either $\alpha_{0}$ or $\alpha_{1}$. We will write eq $70 \mathrm{a}$ in two ways as

$$
\begin{aligned}
\mathrm{d}_{2} r & =S_{1} \alpha_{0}+S_{2} \\
& =S_{3} \alpha_{1}+S_{4}
\end{aligned}
$$

where $S_{\mathrm{i}}$ is given as

$$
\begin{aligned}
S_{1}= & b K_{21} \\
S_{2}= & \left(1-d_{1}^{-1} d_{2}\right) \rho_{30} V_{0} w_{0} \\
S_{3}= & b K_{21} k_{21} b_{2}^{-1} \\
& \left.-\left(1-d_{1}^{-1} d_{2}\right) v_{1}\right] \rho_{30} V_{0} . \\
S_{4}= & -\left[\left(d_{1}^{-1} d_{2}-k_{0} b_{2}^{-1}\right)\left(w_{0}-v_{1}\right)\right.
\end{aligned}
$$

We will examine the value of $S_{i}$ for a special case in which $\sigma$ vanishes and $V_{0}$ is much less than $f_{10}$. The limiting value of $S_{\mathrm{i}}$ as $V_{0}$ approaches zero is given as

$$
\begin{aligned}
& S_{1}=b^{*} K_{21}^{*} \\
& S_{2}=0 \\
& S_{3}=b^{*} K_{21}^{*} S \\
& S_{4}=0 \\
& S=k_{21}\left(k_{0}+L b^{*} K_{21}^{*}\right)^{-1} .
\end{aligned}
$$

It follows from eq71a, 71b,71c and 71d that the last term on the right-hand side of eq $70 b$ (or 70c) is negligible in comparison with the first term in the right-hand side of eq $70 \mathrm{~b}$ (or 70c) when $V_{0}$ is much less than $f_{10}$ and $\alpha_{\mathrm{i}} \geq 1.0^{\circ} \mathrm{C} \mathrm{cm}^{-1}$ for $i=1,2$. It is easy to find that $S_{1}, S_{2}$ and $S_{3}$ are positive, but the sign of $S_{4}$ is not certain.

According to the results of our analysis, we have found that the rate of frost heave $r$ is not uniquely determined by the speed of a frost front $V_{0}$ alone and that the relationship between $r$ and $V_{0}$ strongly depends on $\alpha_{i}(i=0,1)$. To examine the validity of eq $70 \mathrm{~b}$ we will use reported experimental data. Takashi et al. (1978) conducted a series of frost heave tests in which the temperature of the unfrozen part $R_{0}$ was kept constant at $0.2-0.3^{\circ} \mathrm{C}$ higher than the freezing point of a sample so that the speed $V_{0}$ of a frost front $n_{0}$ was kept nearly constant. Dividing eq $70 \mathrm{~b}$ by $d_{2} V_{0}$, we will reduce eq $70 \mathrm{~b}$ to

$$
\begin{aligned}
\hat{\xi}= & r V_{0}^{-1}=d_{2}^{-1} b K_{21} \alpha_{0} V_{0}^{-1} \\
& +\left(d_{2}^{-1}-d_{1}^{-1}\right) \rho_{30} w_{0} .
\end{aligned}
$$

Takashiet al.(1978) called $\hat{\xi}$ the "frostheave ratio." Analyzing their data, Takashiet al. (1978) found empirically that $\hat{\xi}$ is uniquely determined by $V_{0}$ for a given applied pressure $\sigma$. A typical behavior of $\hat{\xi}$ vs. $V_{0}$ obtained by them is reproduced in Figure 4 where a curve is drawn that approximately represents their data points taken with their sample 2 under the applied pressure $\sigma=304 \mathrm{kPa}$. In their tests the temperature profile in the sample was not measured and it is difficult to assess the variability of $\alpha_{0}$. However, if $\alpha_{0}$ is kept nearly constant and $K_{21}$ mainly depends on $\alpha_{0} V_{0}^{-1}$, then their empirical relationships between $\hat{\xi}$ and $V_{0}$ are consistent with eq 72 as we will show below.

Since the second term on the right side of eq 72 is a given constant, $\hat{\xi}$ approaches asymptotically this constant as $V_{0}$ becomes infinite. The value of $\hat{\xi}$ increases with the decreasing $V_{0}$ until $\hat{\xi}$ becomes infinite when $V_{0}$ vanishes and an ice layer grows. 


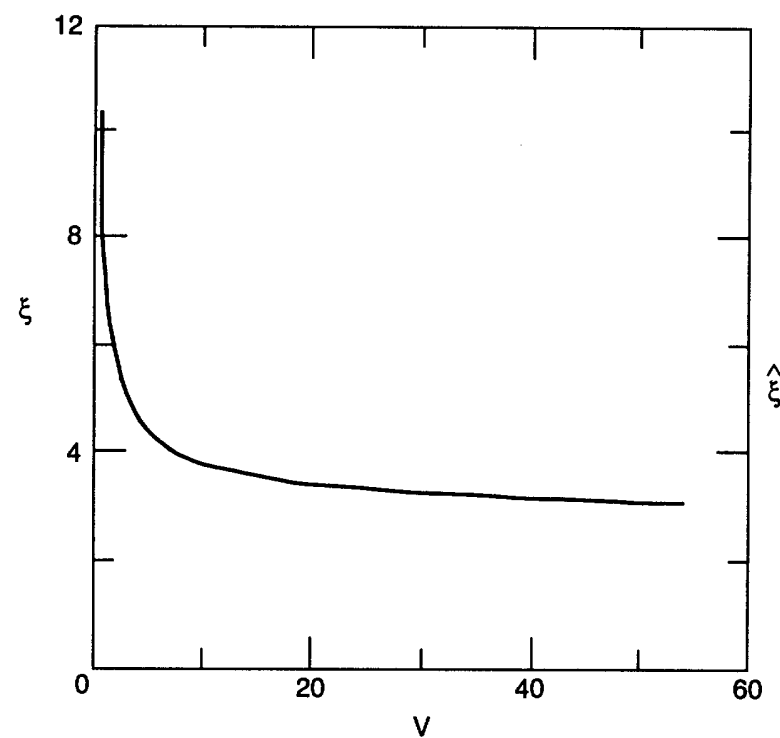

Figure 4. Frost heave ratio $\hat{\xi}(\%)$ vs. $\mathrm{V}_{0}\left(\mathrm{~cm} \mathrm{~d} d^{-1}\right)$ obtained empirically by Takashi et al. (1978).

The curve $\hat{\xi}$ vs. $V_{0}$ in Figure 4 is converted into the curve $\hat{\xi}$ vs. $V_{0}^{-1}$ in Figure 5 . It is clear from eq 72 that the gradient of the curve in Figure 5 is proportional to $K_{21}$ if eq72holds true. From Figure 5 we find that the gradient of the curve tends to decrease with the increasing $V_{0}^{-1}$; namely, $K_{21}$ is a decreasing function of $V_{0}^{-1}$.

We have derived eq 72 under the assumption that the speed of a frost front $V_{0}$ is constant. Therefore, eq 72 is not anticipated to hold true for the transient freezing in which $V_{0}$ varies with time. However, eq 72 may approximately hold true for the transient case in which the change of $V_{0}$ with time is small. Analyzing the data on transient freezing tests obtained by Akagawa (1990), Miyata and Akagawa (1991) empirically found that $\hat{\xi}$ may be uniquely determined by $\alpha_{o} V_{0}^{-1}$, though data are limited. Their data $\hat{\xi}$ of two tests (test $A$ with $\sigma=60$ $\mathrm{kPa}$ and test $\mathrm{B}$ with $\sigma=110 \mathrm{kPa}$ ) vs. $\alpha_{o} V_{0}^{-1}$ are presented in Figure 6 where a curve is drawn to show the trend of the data points.

A soil specimen of $8.5-\mathrm{cm}$ length was frozen from the bottom up with constant boundary temperatures (Akagawa 1990). The data points $(\hat{\xi}$, $\alpha_{o} V_{0}^{-1}$ ) were taken during the time period from 4 to 45 hours after the start of the test. The speed $V_{0}$ decreased and $\alpha_{0}$ increased monotonically with time; hence, the value $\alpha_{o} V_{0}^{-1}$ increased monotonically with time. In test $\mathrm{A}$, for instance, $V_{0}$ changed from $9.12 \mathrm{~cm} \mathrm{~d}^{-1}$ at 4 hours to nearly zero at 45 hours while $\alpha_{0}$ changed from about $0.6^{\circ} \mathrm{C} \mathrm{cm}^{-1}$ at 4 hours to about $1.5^{\circ} \mathrm{C} \mathrm{cm}^{-1}$ at 23 hours. It is quite interesting that eq 72 may hold true despite such

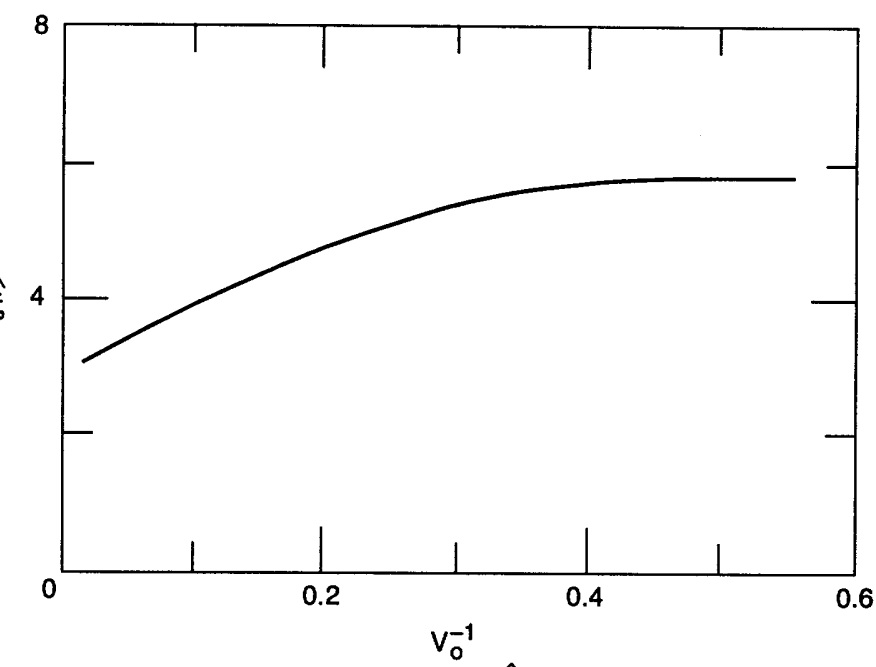

Figure 5. Frost heave ratio $\hat{\xi}(\%)$ vs. $\mathrm{V}_{0}^{-1}\left(\mathrm{~cm}^{-1} d\right)$ obtained empirically by Takashi et al. (1978).

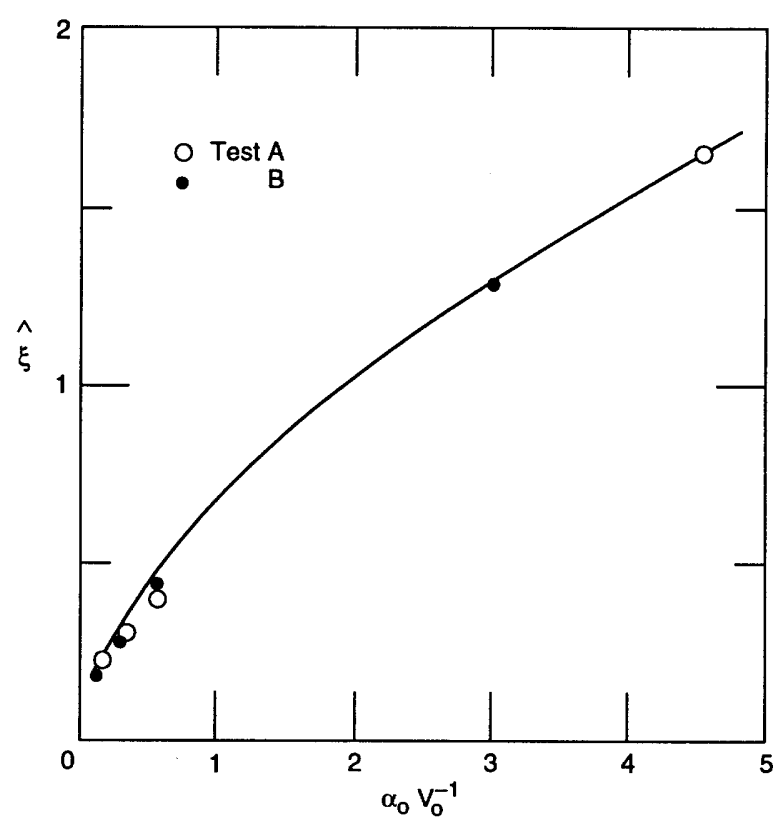

Figure 6. Frost heave ratio $\hat{\xi}(\%)$ vs. $\alpha_{0} \mathrm{~V}_{0}^{-1}\left({ }^{\circ} \mathrm{Ccm}^{-2} d\right)$ obtained empirically by Miyata and Akagawa (1991).

rates of change in $V_{0}$ and $\alpha_{0}$ as described above. From Figure 6 we find a trend similar to that of Figure 5: the gradient of the curve $\hat{\xi}$ vs. $\alpha_{o} V_{0}^{-1}$ tends to decrease with the increasing $\alpha_{o} V_{0}^{-1}$, namely, $K_{21}$ is a decreasing function of $\alpha_{o} V_{0}^{-1}$.

We have studied the steady growth of ice-rich frozen soil by using $M_{1}$. We have shown that there exists a traveling wave solution to the problem of steadily growing ice-rich frozen soil and that this solution is reduced to the solution to the problem 
of a steadily growing ice layer when the velocity $\dot{n}_{0}$ vanishes. We have also shown that the steady growth condition of ice-rich frozen soil under given hydraulic conditions and applied pressures is uniquely determined by a set of two physical variables, $\alpha_{0}$ and $\alpha_{1}$. We will present the results of our experimental study in another report.

\section{LITERATURE CITED}

Akagawa, S.(1990)X-ray photography method for experimental studies of the frozen fringe characteristics of freezing soil. USA Cold Regions Research and Engineering Laboratory, Special Report 90-5.

Edlefsen, N.E. and A.B.C. Anderson (1943) Thermodynamics of soil moisture. Hilgardia, 15(2): 31298.

Miyata, Y. and S. Akagawa (1991) Factors governing a frost heave ratio. In Proceedings, 6th International Symposium on Ground Freezing, Beijing, Chi$n a$, vol. 1, p. 55-63.

Nakano, Y. (1986) On the stable growth of segregated ice in freezing soil under negligible overburden pressure. Advances in Water Resources, 9: 223235.

Nakano, Y.(1990)Quasi-steady problems in freez- ing soils: I. Analyses on the steady growth of an ice layer. Cold Regions Science and Technology, 17(3): 207-226.

Nakano, Y. and K. Takeda (1991) Quasi-steady problems in freezing soils: III. Analysis on experimental data. Cold Regions Science and Technology, 19: 225-243.

Radd, F.J. and D.H. Oertle (1973) Experimental pressure studies of frost heave mechanism and the growth-fusion behavior of ice. In Permafrost: The North American Contribution to the 2nd International Conference on Permafrost, Yakutsk, 13-28 July. Washington, D.C.: National Academy of Sciences, p. 377-384.

Sansone, G. and R. Conti (1964) Nonlinear Differential Equations. Oxford: Pergamon Press, p. 13-15. Takashi, T., H. Yamamoto, T. Ohrai and M. Masuda (1978) Effect of penetration rate of freezing and confining stress on the frost heave ratio of soil. In Proceedings, 3rd International Conference on Permafrost, 10-13 July. Edmonton, Alberta. Ottawa: National Research Council of Canada, vol. 1, p. 737742.

Takeda, K. and Y. Nakano (1990) Quasi-steady problems in freezing soils: II.Experiment on growth of an ice layer. Cold Regions Science and Techology, 18: 225-247. 


\section{APPENDIX A: EXACT SOLUTION OF EQUATION 45}

When $k_{1}(\xi)$ is given by eq $40 \mathrm{e}$, we will introduce a new independent variable $X$ defined for $n_{1} \geq \xi \geq n_{0}$ as

$$
X=1+\eta\left(\xi-n_{0}\right), \quad 1+\eta \delta \geq X \geq 1 .
$$

Using $X$, we will reduce eq 45 to

$$
\frac{d T}{d X}=(\eta X)^{-1}\left(\beta_{1} T-\alpha_{0}-k_{0}^{-1} \Lambda L\right) .
$$

Multiplying eq $A 2$ by $X^{-\left(\beta_{1} / m\right)}$, we will write eq $A 2$ as

$$
\frac{d}{d X} T X^{-\left(\beta_{1} / \eta\right)}=-(\eta X)^{-1} X^{-\left(\beta_{1} / \eta\right)}\left(\alpha_{0}+k_{0}^{-1} \Lambda L\right) .
$$

Suppose that a solution $T(X)$ of eq $\mathrm{A} 2$ exists. Since the right side of eq $A 3$ is negative, the function $T X^{-\left(\beta_{1} / \eta\right)}$ is a decreasing function of $X$. Therefore, $T(X)$ must be negative for $X>1$ because $T=0$ at $X=1$. We have found that a solution $T(X)$ is negative for $X>1$ if it exists. Integrating eq $A 2$, we obtain

$$
\ln X=\int_{0}^{T} \eta\left(\beta_{1} T-\alpha_{0}-k_{0}^{-1} \Lambda L\right)^{-1} d T .
$$

Since the integrand of eq A4 is continuous, a solution $T(\mathrm{X})$ exists. It is easy to see that the right side of eq A2 satisfies a Lipschitz condition with respect to $T$ because the function $\Lambda(T)$ possesses a continuous first derivative as assumed. Based on an elementary theorem of ordinary differential equations (Sansone and Conti 1964), we may conclude that eq $A 2$ has a unique negative solution $T(X)$ for $X>1$.

It follows from eq $A 2$ that the unique solution of eq $\mathrm{A} 2$ is decreasing with $x$ (or $\xi$ ) because the right side of eq $A 2$ is negative. We have shown that eq 45 has a unique and decreasing solution for $n_{1} \geq \xi \geq n_{0}$. 


\section{APPENDIX B: APPROXIMATE SOLUTION OF EQUATION 45}

As we have shown above (App. A), eq 45 has a unique and decreasing solution. This implies that $X$ (or $\xi$ ) and $T$ are one-to-one. Treating $\Lambda$ as a function of $X$, we will write eq $\mathrm{A} 2$ as

$$
T(X)=\pi_{1} \beta_{1}^{-1}\left(1-X^{\beta_{1} \eta^{-1}}\right)+\pi_{0} \eta^{-1} Y X^{\beta_{1} \eta^{-1}}
$$

where $\pi_{0}, \pi_{1}$, and $Y$ are defined as

$$
\begin{aligned}
& \pi_{0}=k_{0}{ }^{-1} L \rho_{30} V_{0} \\
& \pi_{1}=\alpha_{0}+\pi_{0} w_{0} \\
& Y(X)=\int_{1}^{X} \vee X^{-\left(1+\beta_{1} \eta^{-1}\right)} d X .
\end{aligned}
$$

We will rewrite eq $\mathrm{A} 2$ as

$$
\frac{d T}{d X}=-(\eta X)^{-1} \pi_{1} Y_{1}
$$

where $Y_{1}$ is defined as

$$
Y_{1}=1-\pi_{1}^{-1}\left(\beta_{1} T+\pi_{0} v\right) .
$$

Using eq $B 5$, we will reduce eq $B 4$ to

$$
\begin{aligned}
& Y=\eta \pi_{1}^{-1} \bar{v} \\
& \bar{v}=-\int_{0}^{T} v X^{-\beta_{1} \eta^{-1}} Y_{1}^{-1} d T .
\end{aligned}
$$

Using eq $B 7$, we will reduce eq $B 1$ to:

$$
T(\xi)=\pi_{1} \beta_{1}^{-1}\left(1-X^{\beta_{1} \eta^{-1}}\right)+\pi_{0} \pi_{1}^{-1} \bar{v} X^{\beta_{1} \eta^{-1}} .
$$

Using eq B5 and B9, we obtain:

$$
T^{\prime}(\xi) X=-X^{\beta_{1} \eta^{-1}}\left(\pi_{1}-\pi_{0} \pi_{1}^{-1} \beta_{1} \bar{v}\right)+\pi_{0} v . \quad(B 10)
$$

We will seek approximate equations for eq B9 and B10 when the following condition holds true:

$$
\eta \delta<1 \quad \text { and } \quad \beta_{1} \delta<1
$$

We found that the condition, eq B11, holds true when the steady growth of an ice layer occurs (Takeda and Nakano 1990). When eq B1 1 holds true, we may use an approximation (Nakano 1990) given as:

$$
X^{\beta_{1} n^{-1}}=1+\beta_{1}\left(\xi-n_{0}\right) .
$$

Using eq $B 12$, we will reduce eq $B 9$ and $B 10$ to

$$
\begin{aligned}
T(\xi)=\pi_{0} & \pi_{1}^{-1} \bar{v}-\left(\pi_{1}-\pi_{0} \pi_{1}^{-1} \bar{v} \beta_{1}\right)\left(\xi-n_{0}\right)(\mathrm{B} \\
T(\xi) X= & -\pi_{1}+\pi_{0}\left(v+\pi_{1}^{-1} \beta_{1} \bar{v}\right) \\
& -\beta_{1}\left(\pi_{1}-\pi_{0} \pi_{1}^{-1} \beta_{1} \bar{v}\right)\left(\xi-n_{0}\right) .
\end{aligned}
$$

For the calculation of $\bar{v}, X^{-\beta_{1} \eta^{-1}}$ is approximated by using eq $\mathrm{B} 12$ as

$$
X^{-\beta_{1} n^{-1}}=\left[1+\beta_{1}\left(\xi-n_{0}\right)\right]^{-1} .
$$

When $V_{0}$ is very small, from eq $\mathrm{B} 13 \mathrm{~T}(\xi)$ is given as

$$
T(\xi)=-\pi_{1}\left(\xi-n_{0}\right)
$$

Substituting eq B16 into eq B15 we obtain

$$
X^{-\beta_{1} n^{-1}}=\left(1-\beta_{1} \pi_{1}^{-1} T\right)^{-1} .
$$

Now $\bar{v}$ is approximated as

$$
\bar{v}=-\int_{0}^{T} v\left[Y_{1}\left(1-\beta_{1} \pi_{1}^{-1} T\right)\right]^{-1} d T .
$$




\section{APPENDIX C: COMPUTATION OF $\mathrm{I}_{1}$}

Using eq $B 5$, we will reduce eq $56 \mathrm{~b}$ to

$$
-\pi_{1} K_{10} I_{1}=\int_{0}^{T} X\left(K_{10} / K_{1}\right) Y_{1}^{-1} d T .
$$

The term $\beta_{1} T$ in eq $B 6$ describes the effect of sensitive heat that is much less than $\pi_{1}$. Also the term $\pi_{1}^{-1} \pi_{0} v$ in eq $B 6$ is less than one. Therefore, the term $\pi_{1}^{-1}\left(\beta_{1} T+\pi_{0} v\right)$ is generally less than one and we may approximate $Y_{1}^{-1}$ as

$$
Y_{1}^{-1}=1+\pi_{1}^{-1}\left(\beta_{1} T+\pi_{0} v\right) .
$$

Using eq $55 b, 55 c$ and $C 2$, we will reduce eq $C 1$ to:

$$
-\pi_{1} K_{10} I_{1}-w_{o} \pi_{0} \pi_{0}^{-1} T_{1} \phi_{21}
$$$$
=\int_{0}^{T} \dot{\phi}_{1} T_{1}\left(1+\pi_{1}^{-1} \beta_{1} T\right) d T
$$$$
=\phi_{11} T_{1}(1-\varepsilon)
$$

where

$$
\begin{aligned}
& \dot{\phi}_{1}=d \phi_{1} / d T \\
& \varepsilon=\beta_{1} \pi_{1}^{-1}\left(-T_{1}+\phi_{11}^{-1} \int_{0}^{T_{1}} \phi_{1} d T\right)
\end{aligned}
$$




\section{REPORT DOCUMENTATION PAGE}

Public reporting burcen for this collection of information is estimated to average 1 hour per response, including the time for reviewing instructions, searching existing data sources, gathering and maintaining the data needed, and completing and reviewing the collection of information. Send comments regarding this burden estimate or any other aspect of this collection of information. including suggestion for reducing this burden, to Washington Headquarters Services, Directorate for Information Operations and Reports, 1215 Jefferson Davis Highway, Suite 1204, Arlington, VA 22202-4302, and to the Office of Management and Budget, Paperwork Reduction Project (0704-0188), Washington, DC 20503.

\begin{tabular}{|l|l|l|}
\hline 1. AGENCY USE ONLY (Leave blank) & $\begin{array}{c}\text { 2. REPORT DATE } \\
\text { March } 1994\end{array}$ & 3. REPORT TYPE AND DATES COVERED
\end{tabular}

\begin{tabular}{|l|l}
\hline 4. TITLE AND SUBTITLE & 5. FUNDING NUMBERS
\end{tabular}

Traveling Wave Solutions to the Problem of

PE: 6.11.02A

Quasi-Steady Freezing of Soils

6. AUTHORS

Yoshisuke Nakano

PR: 4A161102AT24

TA: SC

WU: F01

7. PERFORMING ORGANIZATION NAME(S) AND ADDRESS(ES)

8. PERFORMING ORGANIZATION REPORT NUMBER

U.S. Army Cold Regions Research and Engineering Laboratory

72 Lyme Road

Hanover, New Hampshire 03755-1290

CRREL Report 94-3

9. SPONSORING/MONITORING AGENCY NAME(S) AND ADDRESS(ES)

10. SPONSORING/MONITORING

AGENCY REPORT NUMBER

Office of the Chief of Engineers

Washington, D.C. 20314-1000

11. SUPPLEMENTARY NOTES

12a. DISTRIBUTION/AVAILABILITY STATEMENT

12b. DISTRIBUTION CODE

Approved for public release; distribution is unlimited.

Available from NTIS, Springfield, Virginia 22161.

13. ABSTRACT (Maximum 200 words)

The results of mathematical and experimental studies presented in preceding reports clearly show that the model $M_{1}$ accurately describes the properties of a frozen fringe when the steady growth of an ice layer occurs. In this work the steady growth of ice-rich frozen soil is studied by using $M_{1}$. Deriving a traveling wave solution to the problem, we have found that the condition of steady growth of ice-rich frozen soil is uniquely determined by a set of two physical variables, such as $\alpha_{0}$ and $\alpha_{1}$ used earlier, under given hydraulic conditions and overburden pressures and that the traveling wave solution converges to the solution to the problem of a steadily growing ice layer when the velocity of the $0^{\circ} \mathrm{C}$ isotherm relative to the unfrozen part of the soil vanishes.

\begin{tabular}{|c|c|c|c|}
\hline \multirow{2}{*}{$\begin{array}{l}\text { 14. SUBJECT TERMS } \\
\text { Frost heave } \\
\text { Frozen soil }\end{array}$} & \multirow{2}{*}{\multicolumn{2}{|c|}{$\begin{array}{l}\text { Mathematical analysis } \\
\text { Traveling wave solutions }\end{array}$}} & $\begin{array}{l}\text { 15. NUMBER OF PAGES } \\
25\end{array}$ \\
\hline & & & 16. PRICE CODE \\
\hline $\begin{array}{l}\text { 17. SECURITY CLASSIFICATION } \\
\text { OF REPORT }\end{array}$ & $\begin{array}{l}\text { 18. SECURITY CLASSIFICATION } \\
\text { OF THIS PAGE }\end{array}$ & $\begin{array}{l}\text { 19. SECURITY CLASSIFICATION } \\
\text { OF ABSTRACT }\end{array}$ & 20. LIMITATION OF ABSTRACT \\
\hline UNCLASSIFIED & UNCLASSIFIED & UNCLASSIFIED & UL \\
\hline
\end{tabular}

